\title{
Verification and Validation of OpenFOAM for High-Lift Aircraft Flows
}

\author{
Neil Ashton* \\ Department of Engineering Science, University of Oxford, UK \\ Vangelis Skaperdas ${ }^{\dagger}$ \\ BETA-CAE Systems, Thessaloniki, Greece
}

This paper presents a detailed investigation into the performance of the open-source finitevolume CFD code OpenFOAM for complex high-lift aircraft flows. A range of cases are investigated including a zero-pressure gradient flat plate, NACA0012 airfoil at varying angles of attack, the DSMA661 airfoil, NASA HLRM and a JAXA JSM high-lift aircraft model. The final three cases were computed as part of the third AIAA HLPW. For all these the same mesh and turbulence model is used to benchmark against the commercial CFD code STAR-CCM+. The paper shows that OpenFOAM using the SA model matches the lift and drag coefficient within 3\% of the commercial code for all the test cases simulated. For the JAXA JSM high-lift aircraft where experimental data is available, both codes show good agreement at pre-stall angles of attack but fail to capture the location of separation at post-stall angles - even though the global lift appears to be well predicted. Whilst OpenFOAM demonstrated comparable accuracy to a range of CFD codes for these aforementioned test-cases, further work is required to improve the robustness and stability of OpenFOAM for these type of flows.

\section{Nomenclature}

$$
\begin{array}{ll}
C_{p} & =\text { pressure coefficient } \\
C_{f} & =\text { skin-friction coefficient } \\
C_{D} & =\text { drag coefficient } \\
C_{L} & =\text { lift coefficient } \\
C_{M} & =\text { pitching moment coefficient } \\
\eta & =\text { ratio of spanwise coordinate to airplane semi-span } \\
\mathrm{c} & =\text { chord } \\
\mathrm{h} & =\sqrt{1 / N}
\end{array}
$$

\footnotetext{
*Senior Researcher, Department of Engineering Science, Oxford, AIAA Senior member, neil.ashton@eng.ox.ac.uk

${ }^{\dagger}$ Manager CFD Applications, BETA-CAE Systems, vangelis@ beta-cae.com
} 


\begin{tabular}{|c|c|c|}
\hline$\rho$ & $=$ & density \\
\hline$M$ & $=$ & Mach number \\
\hline$N$ & $=$ & volume mesh cell count \\
\hline$v$ & $=$ & kinematic viscosity \\
\hline$p$ & $=$ & pressure \\
\hline $\operatorname{Re}$ & $=$ & $c U_{\infty} / v$ \\
\hline$U_{\infty}$ & $=$ & freestream velocity \\
\hline$T_{\infty}$ & $=$ & freestream static temperature \\
\hline AoA & $=$ & Angle of attack \\
\hline CFD & $=$ & Computational Fluid Dynamics \\
\hline CFL & $=$ & Courant-Friedrichs-Lewy condition \\
\hline CRM & $=$ & NASA Common Research Model \\
\hline DES & $=$ & Detached Eddy Simulation \\
\hline HLCRM & $=$ & NASA High-Lift Common Research Model \\
\hline HLPW & $=$ & High Lift Prediction Workshop \\
\hline JAXA & $=$ & Japan Aerospace Exploration Agency \\
\hline JSM & $=$ & JAXA Standard Model \\
\hline LES & $=$ & Large Eddy Simulation \\
\hline MAC & $=$ & Mean Aerodynamic Chord \\
\hline RANS & $=$ & Reynolds Averaged Navier Stokes \\
\hline SA & $=$ & Spalart-Allmaras Model \\
\hline SST & $=$ & Shear-Stress Transport Model \\
\hline TMR & $=$ & Turbulence Modeling Resource \\
\hline
\end{tabular}

\section{Introduction}

NE of the primary drivers of growth in Computational Fluid Dynamics (CFD) has been the aerospace industry.
Over the past 40 years it has evolved from a useful analysis method to a major design tool. At companies such as Boeing much of the early wing design work is conducted almost exclusively in CFD until integration with the other components [1] e.g high-lift devices. A range of computational techniques, from panel methods to Reynolds Averaged Navier-Stokes (RANS) models are used in various parts of the CFD design process, depending on the competing needs of accuracy and turn-around time. As High-Performance Computing (HPC) facilities become ever cheaper and more available, RANS methods (and later high-fidelity methods such as Wall-Modelled Large-Eddy Simulation (WMLES) 
and hybrid RANS-LES methods) will gradually take over a greater share of the entire aerodynamic tool-kit as the cost savings and flexibility over experimental methods become more viable [2-4].

However at present even though RANS methods are commonly used, this is only true in particular parts of the flight envelope, such as the cruise phase [1]. In high-lift configurations, RANS often fail to capture the correct flow physics, typically failing to capture the separation point and the resulting extent of the separated flow region (as shown in recent AIAA HLPW [5]). Higher-fidelity methods arguably offer the greatest hope of using CFD in these more challenging regions of the flight envelope. The cost of hybrid RANS-LES methods such as DES [6] is unfeasibly high for a design environment at present [7], however the progress of HPC means that this may be now less than 5-10 years away [2].

Many aerospace codes, for reasons of national security and confidentiality are neither open-source nor available to foreign nationals. For this reason, researchers wishing to collaborate openly and globally, must look for open-source codes to develop both the methods and software practices to advance the use of CFD. The aerospace sector is a large user of CFD however other industries such as the automotive and energy sector are also key stakeholders in the future of CFD. Thus any such codes should ideally be numerically flexible enough to solve a range of problem types.

There are a number of open-source CFD codes available, from aerospace focused e.g SU2 [8] to energy (nuclear) focused: Code Saturne [9]. It is not within the scope of this paper to attempt to review all the different open-source CFD codes and whilst there may be other codes that fit the bill of a general purpose CFD code, OpenFOAM [10] is arguably one of the most widely used that meets this requirement. Over the past 15 years it has developed from a university code to one which is used by both major industries and Universities, largely because of its growing user base and the comprehensive set of solvers, turbulence models and meshing capabilities. Its largest perceived weakness is a lack of verification and validation, which is particularly true in the aerospace sector, where it does not have a demonstrated track record of matching the results from standard aerospace codes.

This paper attempts to address this and has the following aims:

- Verify and validate OpenFOAM on a range of simple compressible test cases from the NASA TMR website

- As part of the AIAA HLPW, compute the NASA CRM in High-Lift configuration and compare against a major commercial code: STAR-CCM+

- Compute a second high-lift full aircraft - the JAXA JSM model and compare against experimental data and the commercial code: STAR-CCM+

Future goals are to explore improved RANS and hybrid RANS-LES methods, however this paper focuses purely on the SA [11] model in order to allow comparison against the greatest number of other codes and given its status as one of the widely used turbulence models in the aerospace industry. 


\section{Verification and Validation}

For all the following test cases the computational setup is kept the same other than the boundary conditions, which are specific for each test case. Two CFD codes are used in this study; OpenFOAM [10, 12] and STAR-CCM+ by Siemens.

OpenFOAM is an open-source $\mathrm{C}++$ toolbox that is most commonly used for CFD. It supports arbitrary polyhedral unstructured grids and contains a range of incompressible and compressible solvers. In these simulations a segregated pressure-based solver (rhoPimpleFoam) is used with local-time-stepping to accelerate steady-state convergence - for stability purposes the local CFL was typically kept below five. The pimple approach is an OpenFOAM-specific variant of the PISO [13] approach where outer correction loops are used to improve convergence and stability (i.e looping over a single-time-step a set number of times with under-relaxation). A $2^{\text {nd }}$ order upwind scheme is used for the momentum and turbulent convective fluxes. This scheme was used after exhaustive attempts to achieve convergence with the density-based schemes within OpenFOAM, which coincides with the findings of Nikaido et al. [14]. More detailed information about the range of OpenFOAM solvers and numerical schemes is given in Robertson et al. [15].

STAR-CCM+ is a commercial CFD code, which uses a cell-centered finite volume discretization applied to cells of arbitrary polyhedral shapes and offers a range of available physical models and algorithms for variety of applications. In these simulations a fully implicit compressible density based scheme is used with the Roe scheme for the flux. A CFL number of 5-30 was typically used based upon a balance between convergence and stability and a Green-Gauss scheme with a min-mod limiter was used for the gradient calculations. A 2nd order upwind scheme is used for the momentum and turbulent convective fluxes.

\section{A. Zero-Pressure Gradient Flat Plate}

The primary purpose of this test case is to verify the implementation of the SA turbulence model in OpenFOAM and STAR-CCM+ for a spatially developing boundary layer. As per the NASA TMR website 7 we use the term verification in the context of establishing that the turbulence model equations are implemented correctly and compare against CFL3D and FUN3D that have undergone significant verification of the SA turbulence model. The mesh resolution in this study means that by the finest grid the only differences should be due to implementation and boundary conditions. The setup of the case is shown in Fig. 1(a) and an illustration of the computational mesh and domain is shown in Fig. 1(b) Four quasi-2D meshes are used, each successively containing double the number of cells in each spatial direction $69 \times 49,137 \times 97,273 \times 193$ and $545 \times 385$.

It can be seen from Fig. 2 that both STAR-CCM+ and OpenFOAM agree well with the NASA structured (CFL3D) and unstructured (FUN3D) codes for the skin-friction and drag coefficient, which is in agreement with Gomez et al. [16]. Both codes shown less sensitivity to the mesh than CFL3D and FUN3D which was also seen by Nikaido [14]. The

\footnotetext{
*http://turbmodels.larc.nasa.gov
} 


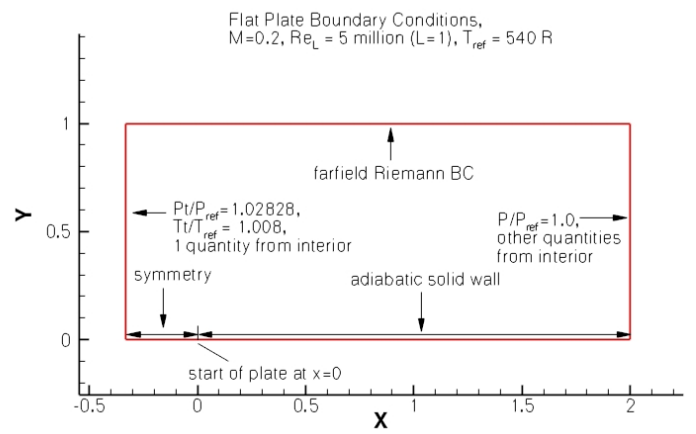

(a)

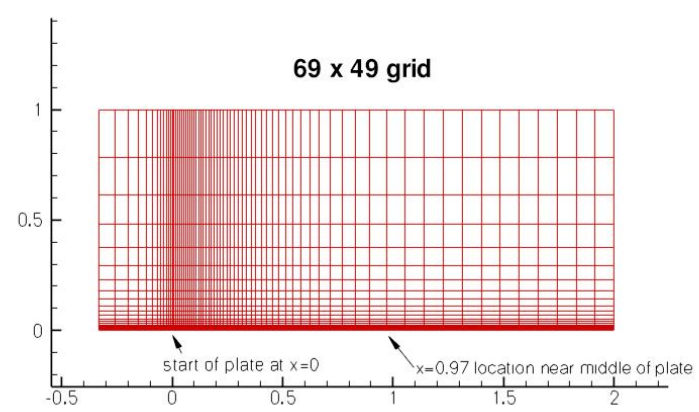

(b)

Fig. 1 Zero-pressure gradient Flat Plate (a) boundary conditions and (b) computational grid and domain (Courtesy of NASA TMR website).

turbulent viscosity ratio is in good agreement between OpenFOAM, CFL3D and FUN3D with STAR-CCM+ having a slightly larger value. Due to the commercial nature of the code, it is not possible to see the exact implementation of the SA model and thus there may be slight differences which cause this increase in the turbulence levels.

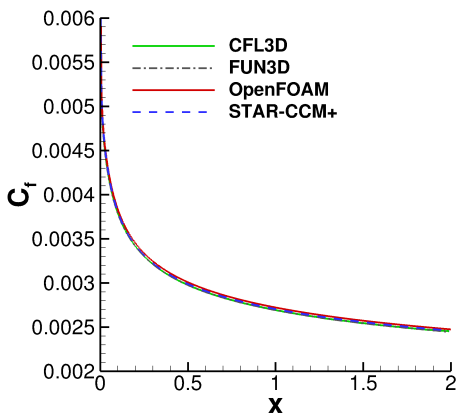

(a)

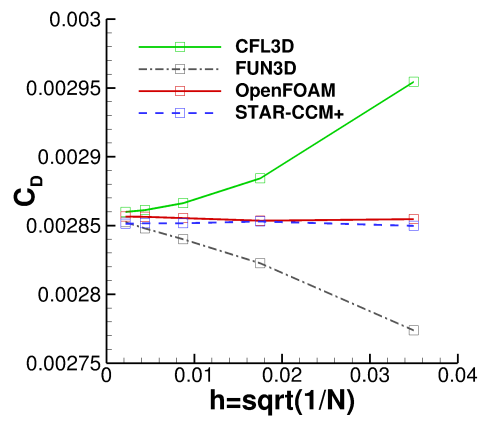

(b)

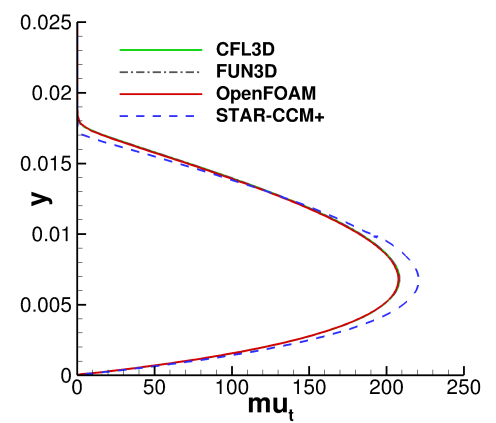

(c)

Fig. 2 Mesh convergence for a zero-pressure gradient Flat plate for FUN3D, CFL3D, OpenFOAM and STAR$\mathrm{CCM}+$ for the (a) Skin-friction coefficient $(545 \times 385$ grid), (b) drag coefficient and (c) turbulent viscosity ratio at $\mathbf{x}=\mathbf{0 . 9 7}$.

\section{B. NACA0012 airfoil}

The NACA0012 airfoil provides a opportunity to assess both codes for a flow exhibiting flow separation. The setup of the case is shown in Fig. 3(a) and an illustration of the computational mesh and domain is shown in Fig. 3(b). Four quasi-2D meshes are used, each successively containing double the number of cells in each spatial direction - $225 \times 65$, $449 \times 129,897 \times 257$ and $1793 \times 513$.

Table 1 shows the lift and drag coefficients for 0,10 and 15 degrees AoA for a range of CFD codes obtained from the 


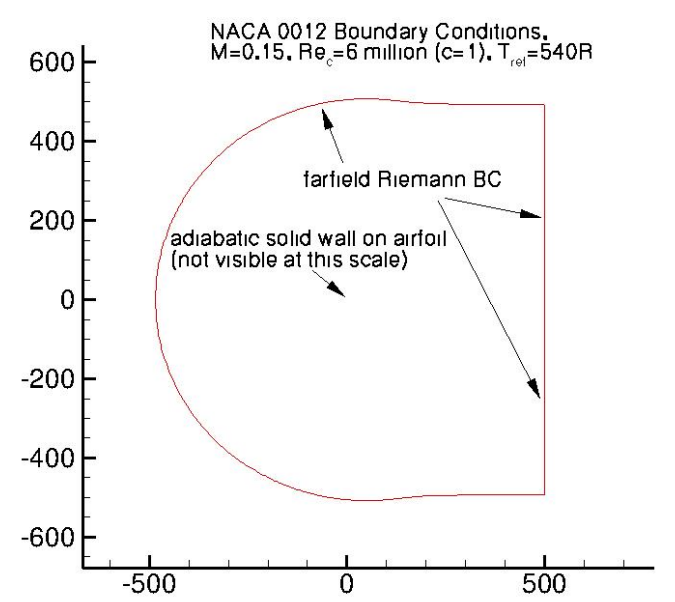

(a)

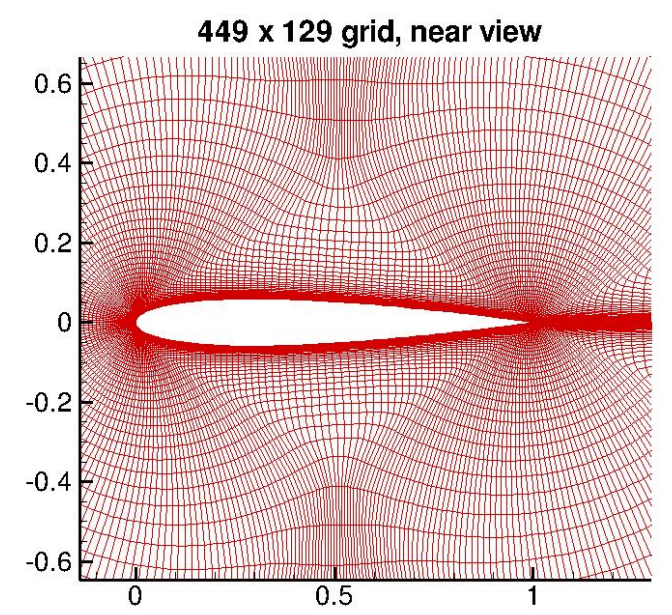

(b)

Fig. 3 NACA0012 airfoil (a) boundary conditions and (b) computational grid and domain (Courtesy of NASA TMR website).

NASA TMR site. It can be seen that OpenFOAM and STAR-CCM+ fall within the range of the other codes which was also shown in Stoellinger et al [17]. However it should be noted that some of these use different versions of the SA model (i.e no-ft2 or SA-neg) and thus likely contribute to the differences observed between these range of codes.

Table 1 Force coefficients for a variety of CFD codes using the $897 \times 256$ grid from the NASA TMR website.

\begin{tabular}{|c|c|c|c|c|c|c|}
\hline \multirow[b]{2}{*}{ Code } & \multicolumn{3}{|c|}{$C_{L}$} & \multicolumn{3}{|c|}{$C_{D}$} \\
\hline & $\alpha=0^{0}$ & $\alpha=10^{0}$ & $\alpha=15^{0}$ & $\alpha=0^{0}$ & $\alpha=10^{0}$ & $\alpha=15^{0}$ \\
\hline CFL3D & $\approx 0$ & 1.0909 & 1.5461 & 0.00819 & 0.01231 & 0.02124 \\
\hline FUN3D & $\approx 0$ & 1.0983 & 1.5547 & 0.00812 & 0.01242 & 0.02159 \\
\hline NTS & $\approx 0$ & 1.0891 & 1.5461 & 0.00813 & 0.01243 & 0.02105 \\
\hline JOE & $\approx 0$ & 1.0918 & 1.5490 & 0.00812 & 0.01245 & 0.02148 \\
\hline SUMB & $\approx 0$ & 1.0904 & 1.5446 & 0.00813 & 0.01233 & 0.02141 \\
\hline TURNS & $\approx 0$ & 1.1000 & 1.5642 & 0.00830 & 0.01230 & 0.02140 \\
\hline GGNS & $\approx 0$ & 1.0941 & 1.5576 & 0.00817 & 0.01225 & 0.02073 \\
\hline OVERFLOW & $\approx 0$ & 1.0990 & 1.5576 & 0.00838 & 0.01251 & 0.02149 \\
\hline OpenFOAM & $\approx 0$ & 1.0903 & 1.5502 & 0.00817 & 0.01221 & 0.02091 \\
\hline STAR-CCM+ & $\approx 0$ & 1.0956 & 1.5511 & 0.008044 & 0.01235 & 0.02112 \\
\hline
\end{tabular}

Figure 4 show the mesh convergence at 10 degrees AoA for CFL3D, FUN3D, OpenFOAM and STAR-CCM+. Each code follows it's own path to mesh convergence, but all trend towards to the same solution, particularly for the drag coefficient. OpenFOAM however stands out as having much larger drag at the coarsest mesh levels, which the authors believe is related to the wall-distance computation. This point will be discussed in greater detail for the next test case.

Figures 5 and 6 show the skin-friction and pressure coefficients for the $897 \times 256$ grid at 0 and 15 degrees AoA. 


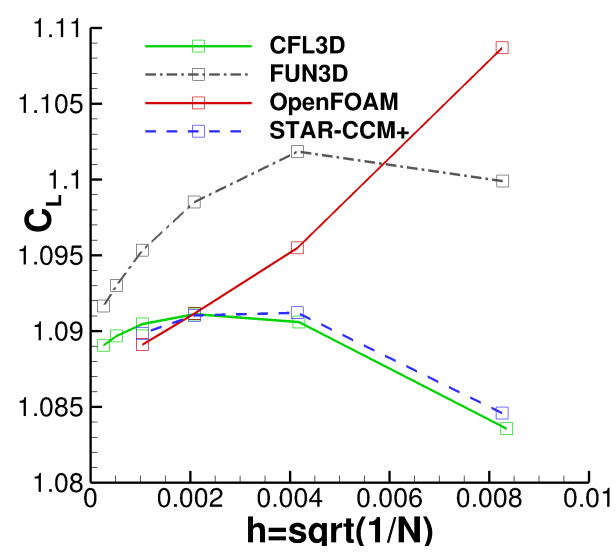

(a)

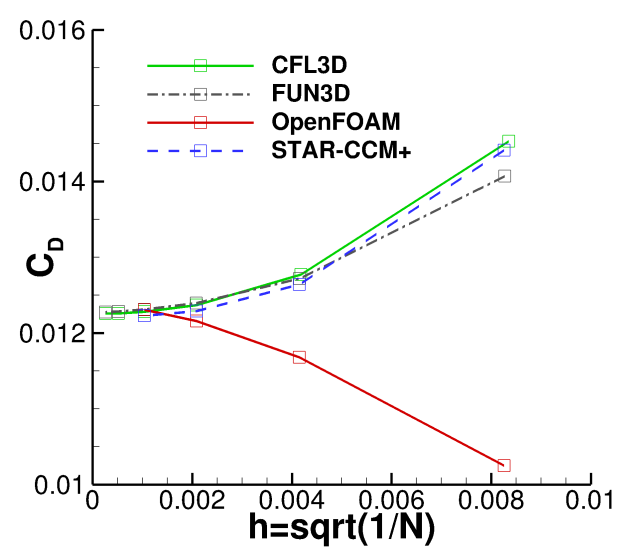

(b)

Fig. 4 Mesh convergence study for the (a) Lift Coefficient and (b) Drag Coefficient at $\alpha=10^{0}$ using the SA model for the NACA0012 airfoil.

There is excellent agreement between both codes at this mesh resolution, which also explains the close agreement for the force coefficients.

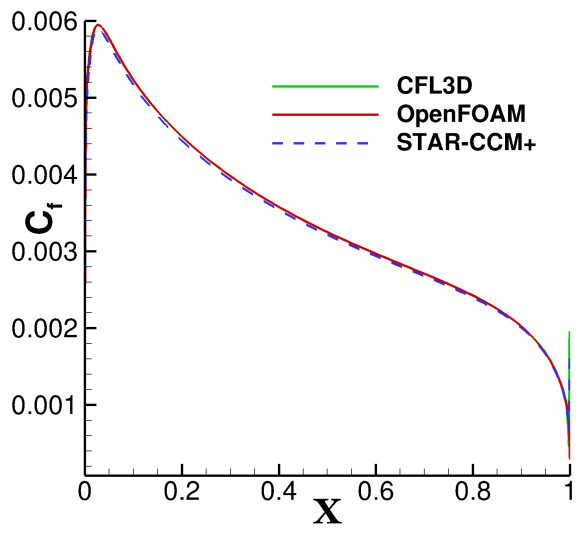

(a)

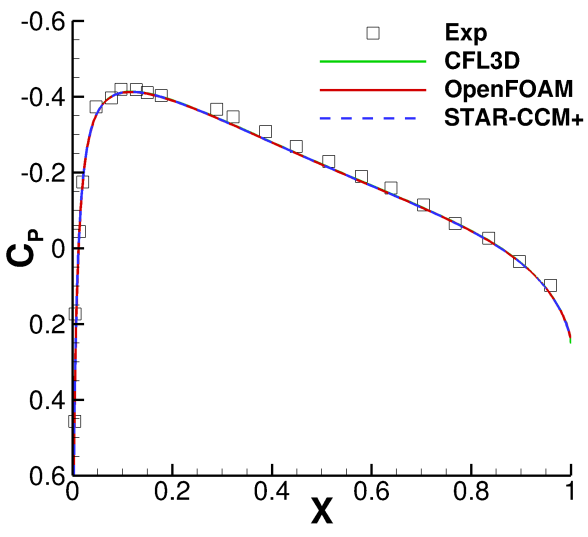

(b)

Fig. 5 (a) Skin Friction Coefficient and (b) Pressure coefficient at $\alpha=0^{0}$ using the SA model in OpenFOAM and STAR-CCM+ for the NACA0012 airfoil.

\section{DSMA661 airfoil}

The DSMA661 airfoil provides another opportunity to assess the implementation of the SA model within OpenFOAM.

The initial setup of the case is shown in Fig. 7(a) and an illustration of the computational mesh and domain is shown in Fig. $7(\mathrm{~b})$ Four grids of $281 \times 49,561 \times 97,1121 \times 193$ and $2241 \times 385$ cells were initially used from the NASA TMR site. 


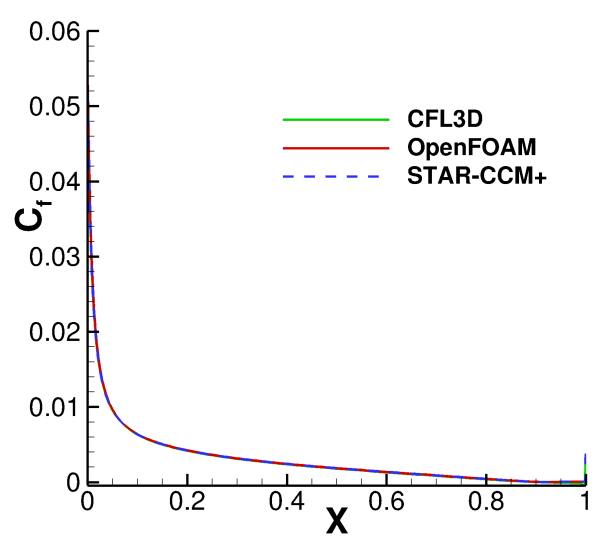

(a)

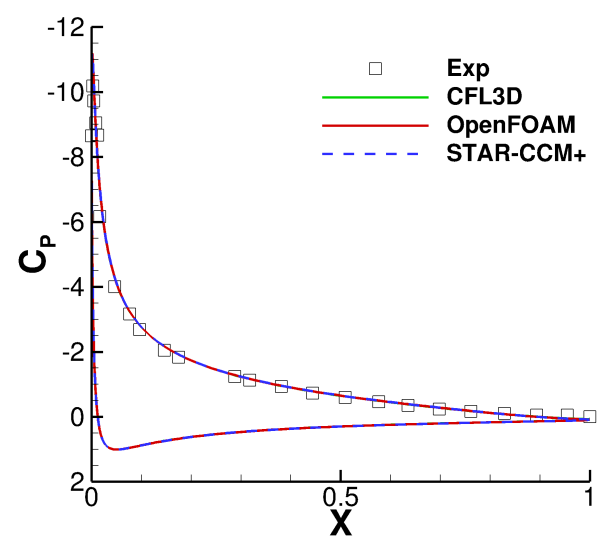

(b)

Fig. 6 (a) Skin Friction Coefficient and (b) Pressure coefficient at $\alpha=15^{0}$ using the SA model in OpenFOAM and STAR-CCM+ for the NACA0012 airfoil.

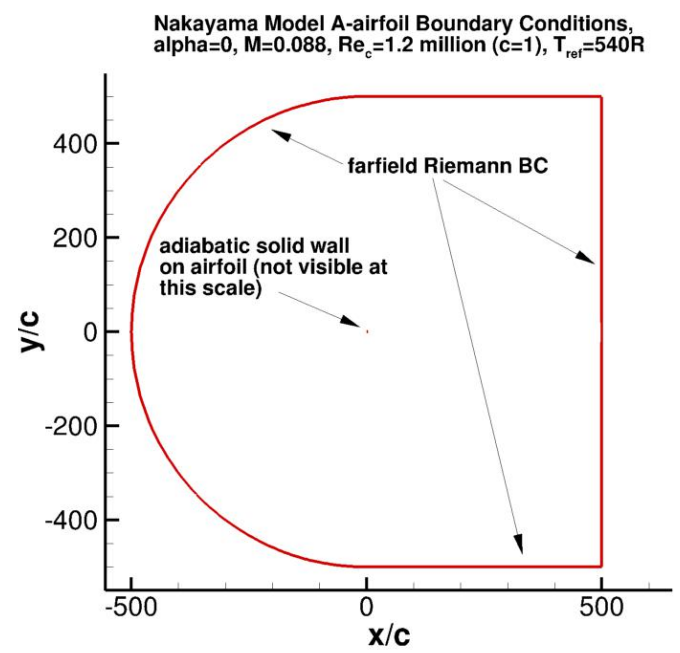

(a)

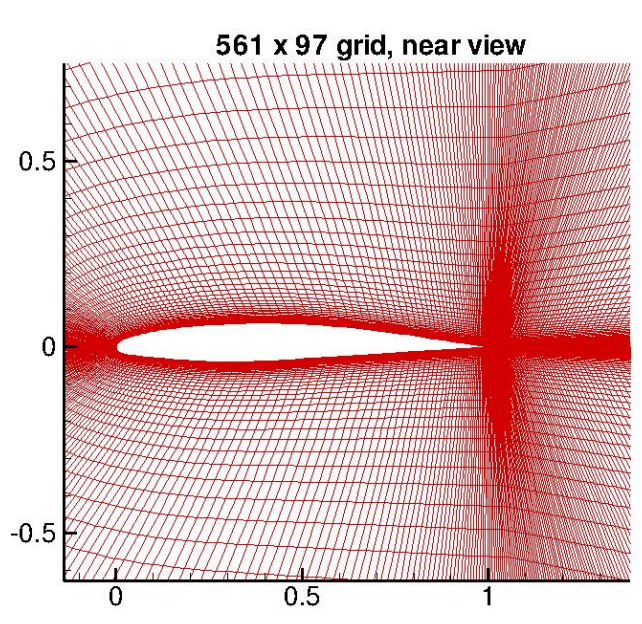

(b)

Fig. 7 DSMA661(MODEL A) airfoil (a) boundary conditions and (b) computational grid and domain (Courtesy of NASA TMR website).

It can be seen in Figs. 8(a) and 8(b) that STAR-CCM+, FUN3D and CFL3D show similar mesh convergence trends for the lift and drag coefficient. For these codes the streamwise velocity (Fig. 8(c)] is also in good agreement with each other and the experimental data by the finest grid.

For OpenFOAM the result on the original mesh from the NASA TMR website shows clearly higher drag and lower lift compared to the other codes. This trend is consistent between grids and thus suggests that numerical dissipation is not causing this inaccuracy. Considering the close agreement between both codes for the flat-plate test case and the 


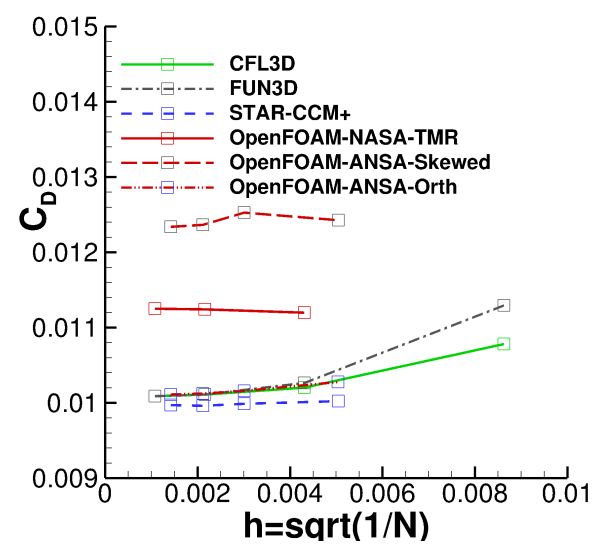

(a)

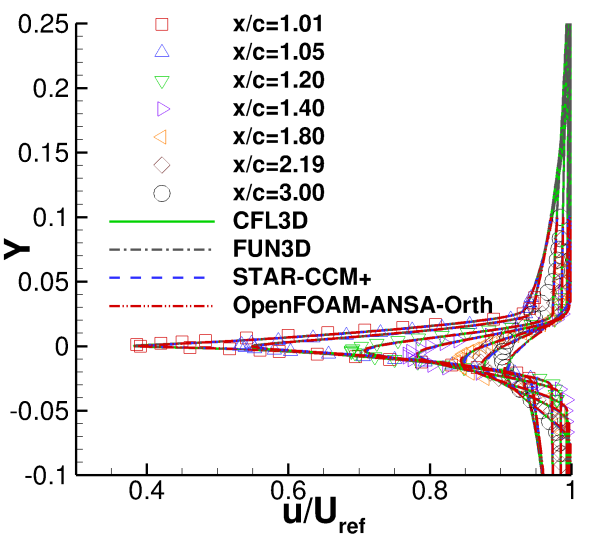

(c)

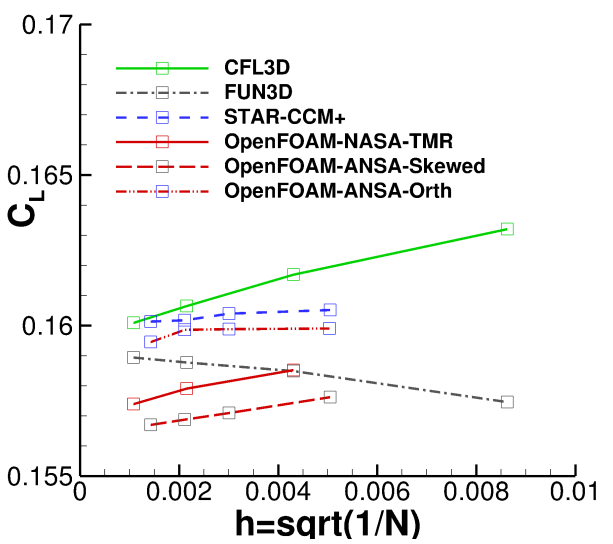

(b)

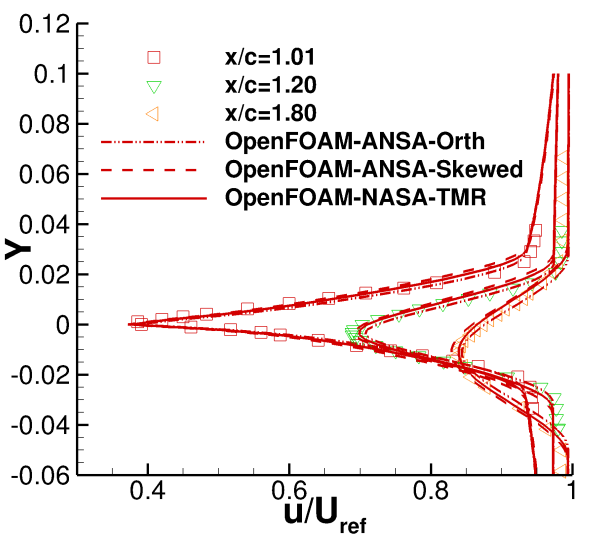

(d)

Fig. 8 (a) Drag Coefficient (b) Lift Coefficient and (c) (d) Streamwise velocity for the DSMA661 (Model A) airfoil.

differing results between OpenFOAM and the other codes for NACA0012, it was concluded that the major difference between the codes was the wall-distance calculation.

For the SA model, the wall-distance $d$, is a key component of the turbulent dissipation term, acting as the turbulent length scale [11].

There are a range of methods to compute the distance to the closest wall, which typically trade off computational cost and accuracy. These range from approximate PDE based approaches [18, 19] to exact KD-Tree search algorithms. A detailed discussion of the wall-distance calculation method within OpenFOAM can be found in Kareem et al [20] but Fig. 9 summarises the different ways the wall distance could be computed for a simple example grid. In Fig. 9 each image shows the same grid, where $C$ is the cell centre and the shaded bottom line is the wall. The shaded cell is the cell where we wish to compute the wall-distance and the two images show how the wall-distance could be computed for a 
different cell location. The wall-distance used in the SA model is defined as the distance from the cell centre to the nearest wall and errors in the calculation of this variable cause errors in $v_{t}$ to grow as $d^{2}$. The default wall-calculation method in OpenFOAM is to compute the nearest wall-face-centre i.e distance from $\mathrm{C}$ to the middle of $\mathrm{A}$ and $\mathrm{B}$. In the first image (Fig. 9(a) this method is close to the exact wall-distance (shown in the blue arrow) with incorrect wall distances being the distance to the nearest wall node (CA, CB). For the second image (Fig. 9(b)) the approach of computing the distance to the middle of the nearest face centre is also incorrect and deviates from the correct distance (C-B shown by the blue arrow). Within OpenFOAM this wall-distance calculation method therefore has the potential to compute the wrong wall-distance, particularly for highly non-orthogonal or skewed grids.

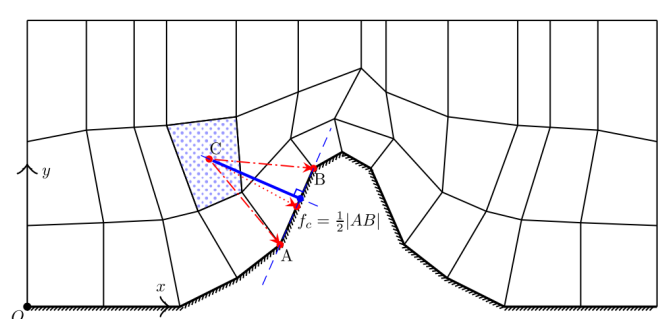

(a)

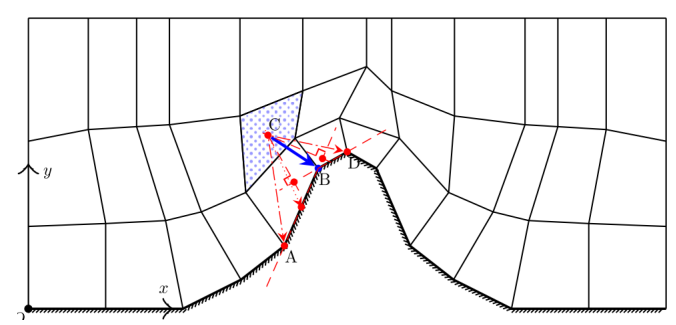

(b)

Fig. 9 Schematic of the different ways to compute the wall-distance for two different cell locations (Figures from Kareem et al. [20] ).

To test the accuracy of the wall-distance computational, two additional grids (shown in Fig. 10) were generated in the mesh generation tool ANSA, using the same surface mesh as the NASA TMR grids. This was done for four refinement levels from $281 \times 49$ to $2241 \times 385$ cells. One grid focused on ensuring orthogonality, as shown in Fig. $10(\mathrm{c})$ and the other created deliberately skewed cells (Fig. 10(b), the reference grid is shown in Fig. 10(a), These were initially ran in STAR-CCM+ to which the result was the same for all three grid families within convergence errors e.g not visible on a graph. For OpenFOAM, the results from the three grid families are shown in Fig. 8 It can be seen that for the grid which ensures orthogonality, the results are in very good agreement with those from the other CFD codes. This is because when the grid is orthogonal, the wall-distance computational is accurate (as shown for the flat-plate test-case). When the grid is skewed, the wall-distance is now incorrect and this results in an over prediction of the drag and an under-prediction of the lift. The NASA TMR site mesh is somewhat between the both, which suggests that the reason OpenFOAM differs from the other code is that the wall-distance calculation is not accurate when the near wall grid is non-orthogonal or skewed.

This idea is re-enforced, given that the SST model, shown in Fig. 11, run on the NASA TMR grids is in close agreement with the reference CFL3D, FUN3D results. For the SST model the wall-distance is only used in the F1 and F2 blending functions (which control the switch between the $k-\varepsilon$ and $k-\omega$ models [21] within the SST model), thus any errors in the wall-distance have less impact on the overall solution. 


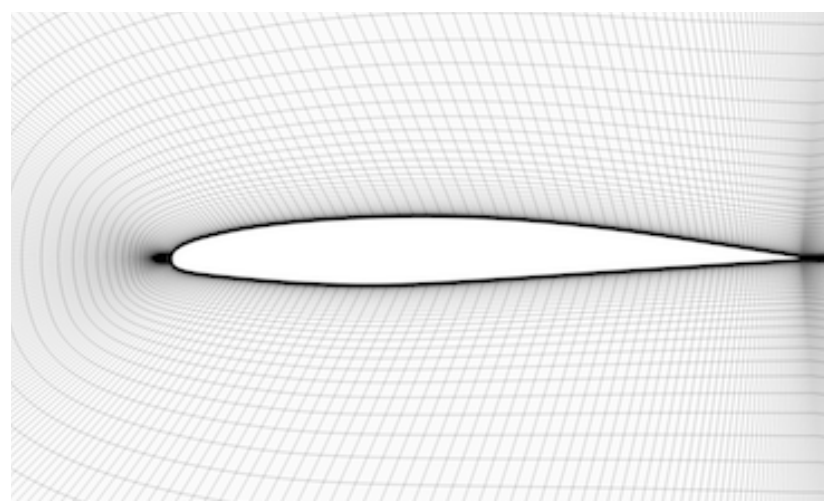

(a)

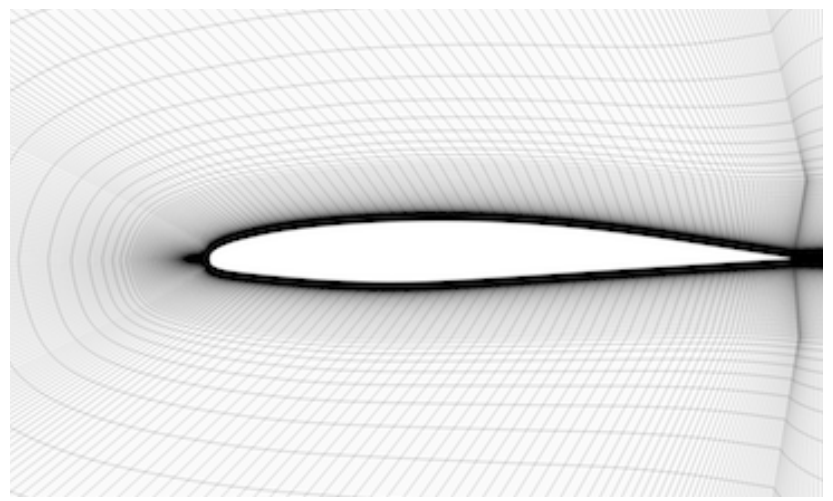

(b)

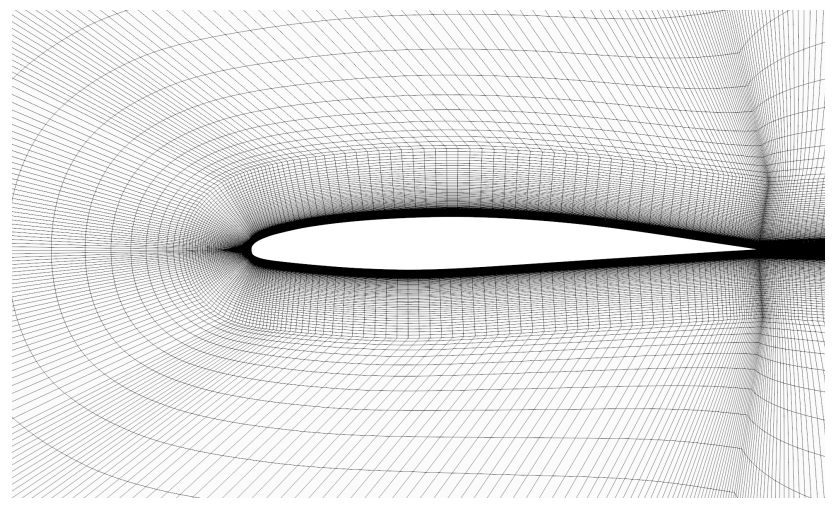

(c)

Fig. 10 Three meshes for the DSMA661(MODEL A) airfoil (a) NASA TMR Grid (b) ANSA Skewed Grid and (c) ANSA Orthogonal Mesh.

Based upon the findings of these validation cases, all the grids used in the study of the high-lift aircraft were designed to have as little non-orthogonality and skewness as possible, to minimise the inaccuracy of the wall-distance computation in OpenFOAM. Future work will be to implement and validate the prototype wall-distance method of Kareem et al [20] into the latest version of OpenFOAM. 


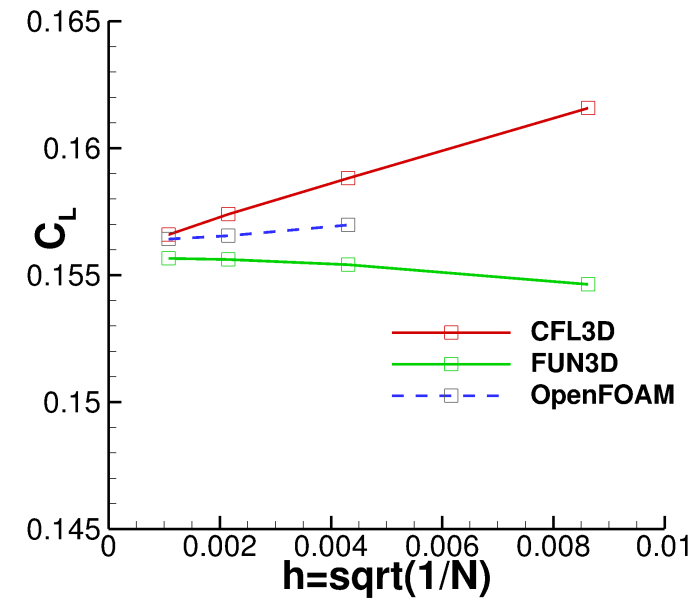

(a)

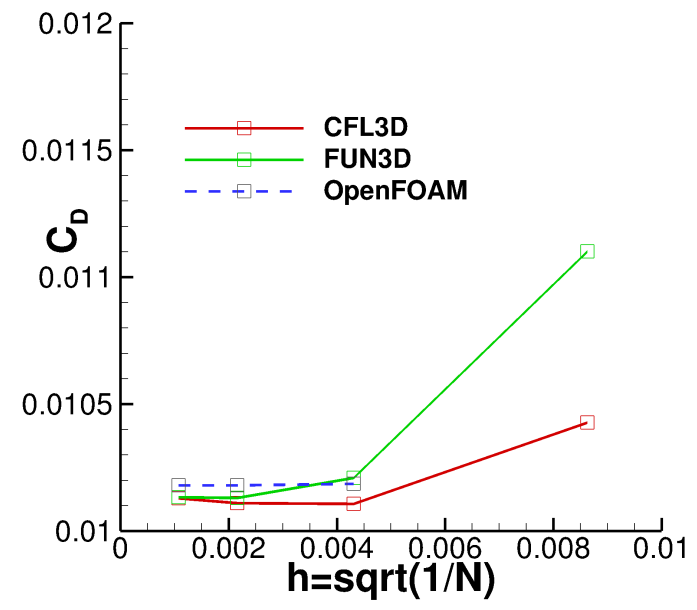

(b)

Fig. 11 (a) Lift coefficient and (b) Drag coefficient for the DSMA661(MODEL A) airfoil using the SST model on the NASA TMR grids.

\section{NASA High-Lift CRM}

In the context of the 3rd AIAA HLPW [22] the NASA CRM in a new high-lift configuration (HL-CRM) [23] is simulated. This is a wing-body high lift system that is studied in a nominal landing configuration (slat and flaps deployed at $30^{\circ}$ and $37^{\circ}$, respectively) without nacelle, pylon, tail, or support brackets. At the time of this publication no experimental data is available, however tests are planned for 2019/2020. The purpose of this test-case in this paper is to assess the accuracy of OpenFOAM for a complex full aircraft geometry. To the best knowledge of the authors, no such geometry has been comprehensively investigated and published using OpenFOAM previously. To provide a benchmark to which OpenFOAM can be judged, simulations are undertaken using the same computational grid in the commercial CFD code STAR-CCM+ v11.6. This is done in a spirit of openness rather than a desire to promote one code over the other. As stated in the introduction, the inability to implement custom turbulence models (among other things) in the majority of commercial codes means that codes like OpenFOAM, SU2 or others are a necessity. STAR-CCM+ was chosen as an example of a mature, widely used code that has been extensively used for compressible high-lift geometries.

\section{A. Computational Set-up}

The HL-CRM is computed in both OpenFOAM and STAR-CCM+. The flow conditions are shown in Table 2 , however as the geometry is full-scale the flow parameters are adjusted to achieve the required Reynolds number. The viscosity is computed using Sutherlands Law and the density is based upon the ideal gas law. Simulations are conducted at $8^{\circ}$ and $16^{\circ}$ angle of attack and all simulations use the SA turbulence model (SA-noft2 according to the NASA TMR website). $2^{\text {nd }}$ order upwind schemes are used for the momentum and turbulent quantities in both OpenFOAM and 
STAR-CCM+.

Table 2 Flow properties for HL-CRM aircraft .

\begin{tabular}{cccccc}
\hline AoA & $M$ & MAC (inches) & $p_{r e f}(P a)$ & $T_{\infty}(\mathrm{K})$ & $R e$ \\
\hline $8^{\circ}$ and $16^{\circ}$ & 0.2 & 275.8 & 101353 & 288 & $3,260,000$ \\
\hline
\end{tabular}

\section{B. Computational Grid}

OpenFOAM has its own mesh generation utility; SnappyHexMesh, a cartesian-prismatic unstructured generation tool, however the experience of the authors has shown that it is not suitable for low $y^{+}$grids and the region between the prismatic and cartesian is often subject to severe non-orthongality and large cell size jumps [24]. For this reason an alternative mesh generator is used, which is capable of generating high-quality grids that represent the kind of unstructured grids that are typically used by the aerospace industry. ANSA 17.1, a pre-processor from BETA-CAE Systems was used to generate all the following grids. The meshing process used for this study is described in detail in Skaperdas et al. [25] thus a brief description is only given here.

The geometry was cleaned up and a hemi-spherical domain of 78,740 inches (285MAC) was created for the half symmetric model. An unstructured triangular surface mesh (Fig. 12) with extruded prisms (and some hexas) and pyramids and tetras in the volume mesh approach was then produced. A factor of 1.5 increase in total cell count was completed twice to form a family or coarse, medium and fine grids.

An important challenge of this work was to generate volume meshes that respected the strict OpenFOAM quality criteria for skewness and non-orthogonality. This is more difficult especially for aerospace type models, where in order to obtain values of $y^{+}$below 1 , the aspect ratio of the elements becomes very large. The problem is more pronounced around areas of sharp corners of the geometry. In order to obtain as good quality criteria elements as possible, an anisotropic mesh strategy was followed in specific areas, like the leading and trailing edges. These areas were generated with map quad mesh with a growth towards the isotropic triangular mesh. This helps in increased resolution along the curvature (at a low cost in cell count) as well as it improves the quality of the extruded top cap of the inflated layers around convex areas. It also improves OpenFOAM skewness as it brings the centroid of the adjacent elements around a corner closer.

Table 3 shows the differing number of layers around the wing and fuselage, which were followed in order to ensure a smooth volume ratio transition between the last extruded layer and the pyramid/tetra mesh. As can be seen in Fig. 13 orthogonality was enforced in the prism layer regions. Around the fuselage, as the surface mesh size is larger, more layers are needed to grow in order to obtain an adequate height and volume of the last layer and connect to the tetra mesh. 


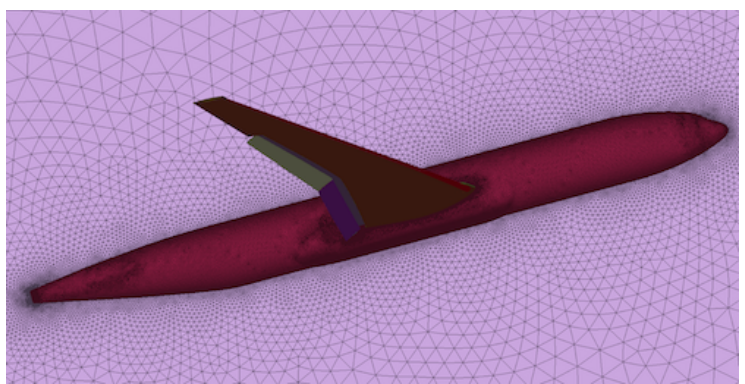

Fig. 12 HL-CRM medium surface mesh.

Table 3 HL-CRM Mesh Properties.

\begin{tabular}{cccc}
\hline & Coarse & Medium & Fine \\
\hline Surface Mesh Face Count & $1.8 \mathrm{M}$ & $2.4 \mathrm{M}$ & $2.8 \mathrm{M}$ \\
Volume Mesh Cell Count & $89 \mathrm{M}$ & $147 \mathrm{M}$ & $236 \mathrm{M}$ \\
Trailing edge rows of elements & 4 & 6 & 8 \\
Number of layers around Wing/Nacelle/Pylon & 40 & 45 & 65 \\
Number of layers around Body & 45 & 57 & 84 \\
Layers Growth & 1.25 & 1.16 & 1.1 \\
Layers First Height (inches) & 0.000787 & 0.000787 & 0.000787 \\
\hline
\end{tabular}

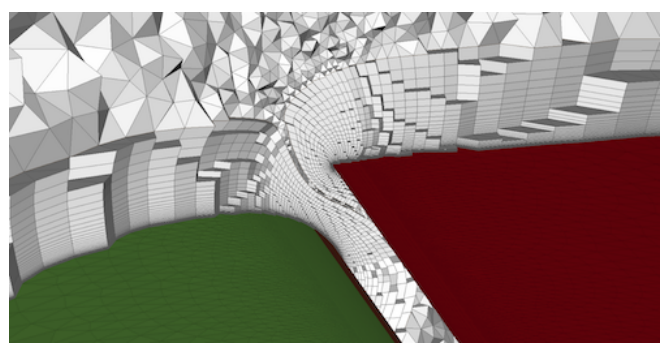

(a)

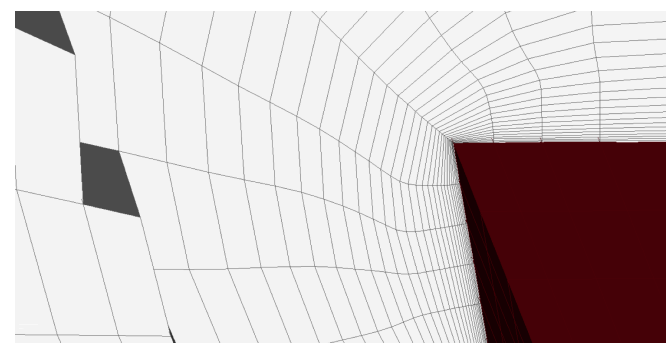

(b)

Fig. 13 Prism layer coverage and details of the imposed orthogonality near the wall for better orthogonality and skewness.

\section{Results}

The first objective of this study was to assess the mesh convergence using a series of three successively finer grids - Coarse, Medium and Fine. Figure 14 shows the mesh convergence of OpenFOAM and STAR-CCM+ for $C_{L}$ and $C_{D}$ at 8 and 16 degrees angle of attack. Several conclusions can be made - firstly that the overall correlation between STAR-CCM+ and OpenFOAM is within 1\% for the Lift coefficient and 3\% for the drag coefficient. Secondly OpenFOAM and STAR-CCM+ trend towards the same mesh converged solution, with OpenFOAM showing a greater sensitivity to the mesh. Finally whilst there is no experimental data for this geometry, the results agree well with other participants running the SA model from the 3rd AIAA HLPW [26]. Even though are only two flow angles simulated, the 
flow doesn't show any in-board separation at the fuselage-wing junction or tip stall with the main separation occurring on the outboard flap. Interestingly the separation actually reduces at the higher angle of attack suggesting that the design of the wing is not well optimized at the lower angle of attack. Further simulations at pre and post stall conditions are needed to investigate the stall mechanisms further.

Whilst the lift and drag coefficients are important quantities, such a global quantity can hide error cancellation. In Figs. 16 and 17 we see a number of planar cuts along the wing-span for the pressure and skin-friction coefficients (the location of these cuts are shown in Fig. 15(a) . For the pressure coefficient the agreement between both codes is very close, with only small differences in the suction peaks. The mesh refinement only made a small change to the pressure coefficient - mainly over the flap, as shown in Fig. 15. For the skin-friction coefficient the agreement is close in the outboard sections but shows some small differences in the inboard section - which may explains the $\approx 3 \%$ difference in the drag coefficient.

In Figs. 18 and 19 the surface streamlines at $\alpha=8^{0}$ and $\alpha=16^{0}$ are shown for OpenFOAM and STAR-CCM+. It can be seen that the flow over the entire wing is very similar between both codes at both angles of attack. For engineering purposes this demonstrates that OpenFOAM can be used for the analysis of this particular configuration of aircraft given that tools such as STAR-CCM+ are used by many engineering companies for major design projects. This also agrees with recent work by the first author on helicopter fuselage geometries [27].

So far however all quantities have been global or surface quantities - in Figs. 21 velocity profiles are shown according to the positions shown in Fig. 20. In a similar vane to the previous quantities, the agreement between STAR-CCM+ and OpenFOAM is close in the off-body region, differing by an account likely within the experimental error range.

\section{JAXA JSM High-Lift Aircraft}

The results from the HL-CRM model have shown excellent agreement between OpenFOAM and STAR-CCM+ for the same turbulence model and mesh. From an engineering perspective, a maximum of 3\% error between the codes is acceptable and suggests that OpenFOAM can be used to analyse these type of flows. At the time of this paper, there is no experimental data available for the HL-CRM, limiting a full discussion of the accuracy of STAR-CCM+ or OpenFOAM. To address this, a further high-lift aircraft model, the JAXA JSM model, is now investigated which includes experimental data from $0^{0}$ to $21^{0}$ AoA [28]. The JSM model is a more realistic aircraft model with slat brackets.

\section{A. Computational Setup}

The flow conditions are shown in Table 4, where the viscosity is computed using Sutherlands Law and the density is based upon the ideal gas law. Simulations are conducted at at $4.36^{\circ}, 10.47^{\circ}, 14.54^{\circ}, 18.58^{\circ}, 20.59^{\circ}$ and $21.57^{\circ}$ angle of

attack and all simulations use the SA turbulence model (SA-noft2 according to the NASA TMR website). $2^{\text {nd }}$ order upwind schemes are used for the momentum and turbulent quantities in both OpenFOAM and STAR-CCM+. 


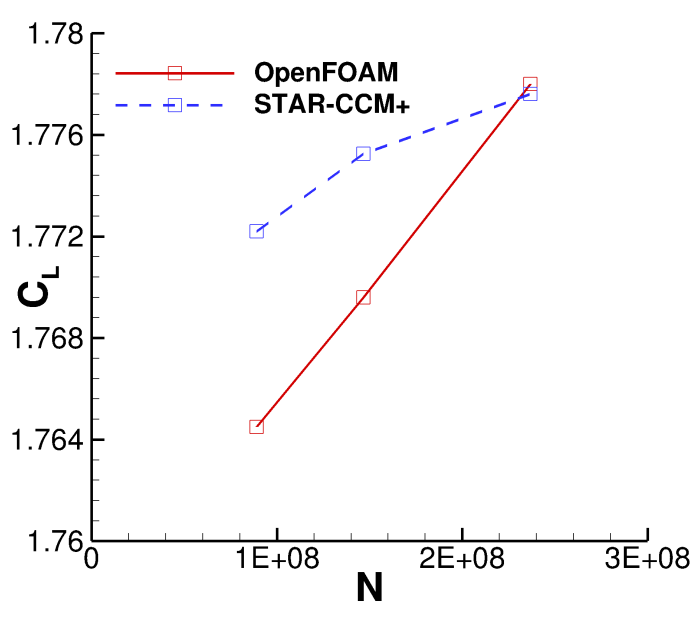

(a)

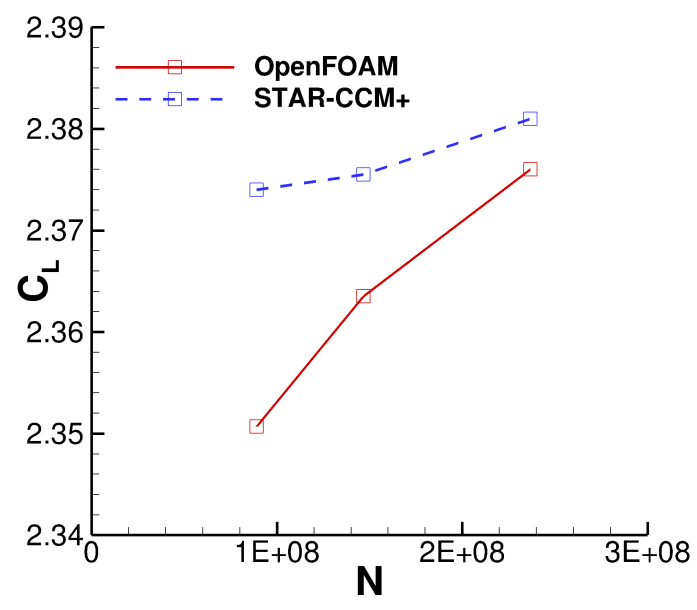

(c)

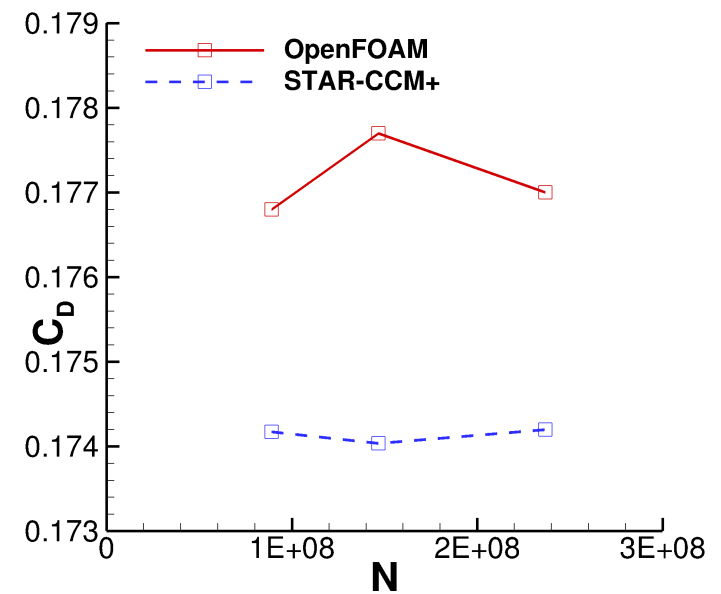

(b)

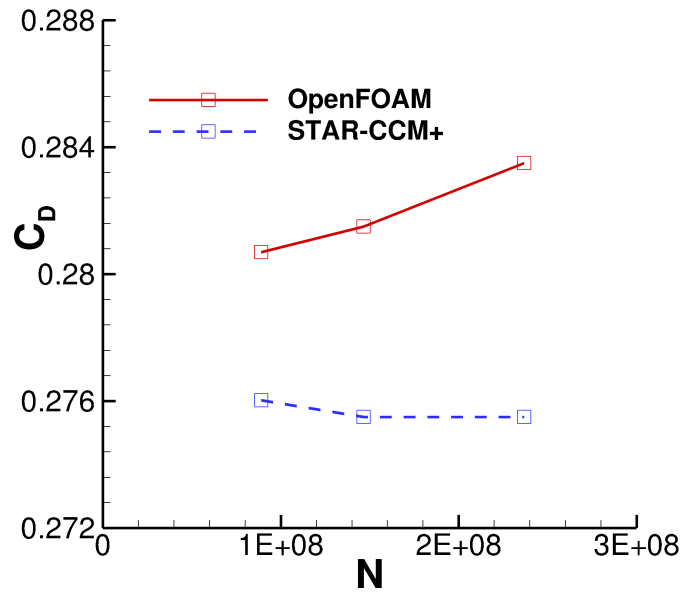

(d)

Fig. 14 Mesh convergence study for the (a) Lift Coefficient and (b) Drag coefficients at $\alpha=8^{0}$ and the (c) Lift Coefficient and (d) Drag Coefficient at $\alpha=16^{\circ}$ for OpenFOAM and STAR-CCM+.

Table 4 Flow properties for JAXA JSM aircraft .

\begin{tabular}{cccccc}
\hline AoA & $M$ & MAC $(\mathrm{m})$ & $p_{\text {ref }}(\mathrm{Pa})$ & $T_{\infty}(\mathrm{K})$ & $\operatorname{Re}$ \\
\hline $4.36^{\circ}, 10.47^{\circ}, 14.54^{o}, 18.58^{\circ}, 20.59^{\circ}, 21.57^{\circ}$ & 0.172 & 0.5292 & 99684 & 306.55 & $1,930,000$ \\
\hline
\end{tabular}

\section{B. Computational Grid}

The meshing tool ANSA v17.1 was again used to generate the computational grid for the JSM geometry. An unstructured triangular surface mesh (Fig. 22) with extruded prisms (and some hexas) and pyramids and tetras in the volume mesh approach was again used. Only a medium mesh (detailed in Table 5] was used in this study, however a fine mesh of a factor of 1.5 increase in total cell count was created and made available to the HLPW participants. 


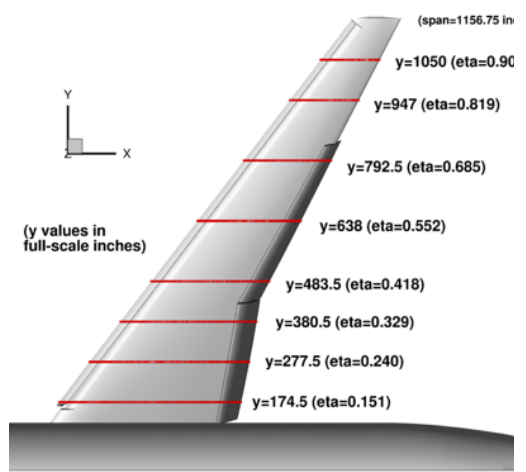

(a)

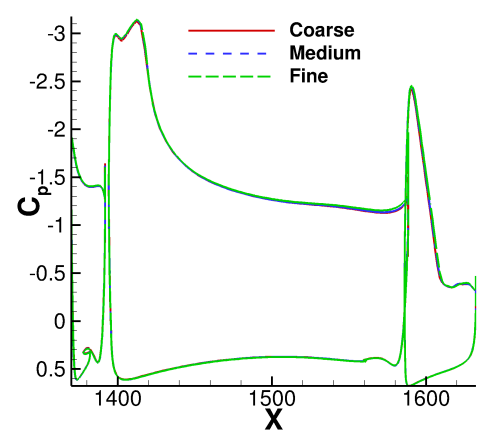

(b)

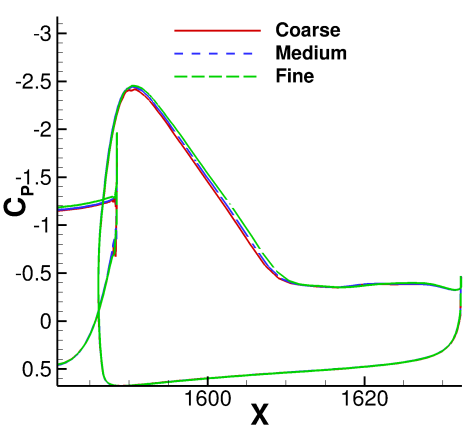

(c)

Fig. 15 (a) Schematic of the planes analysed in this section and (b), (c) mesh convergence of pressure coefficient at eta $=0.552$ for $\alpha=8^{0}$ using OpenFOAM.

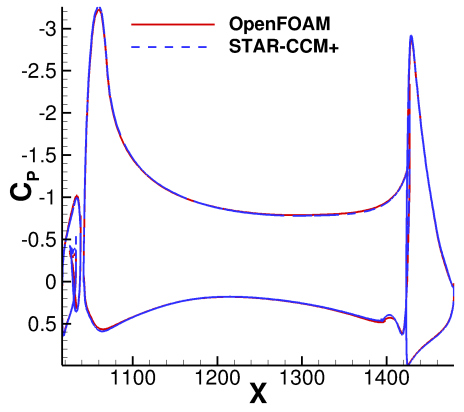

(a)

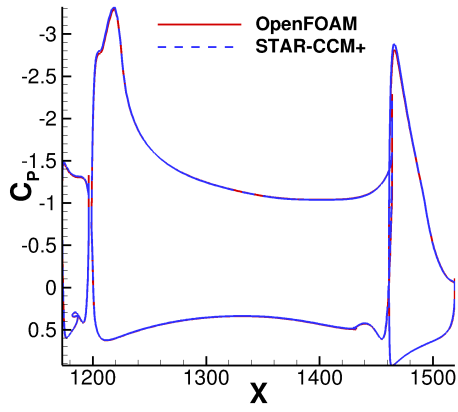

(b)

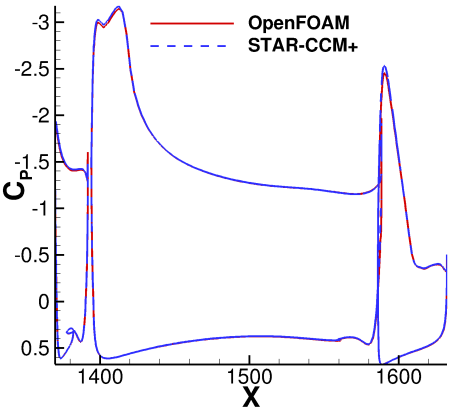

(c)

Fig. 16 Pressure coefficient at (a) eta=0.151, (b) 0.329 and $\left(\right.$ c) 0.552 at $\alpha=8^{0}$ for OpenFOAM and STAR-CCM+.

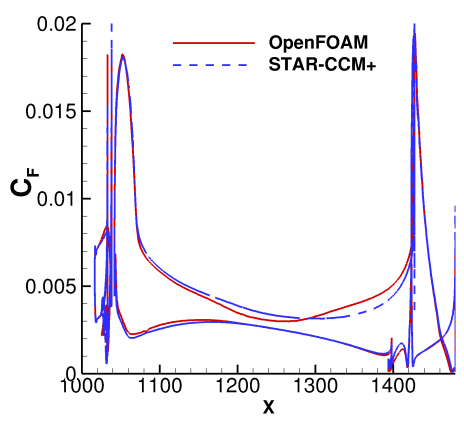

(a)

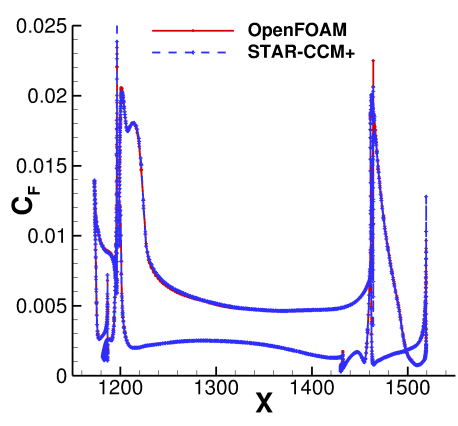

(b)

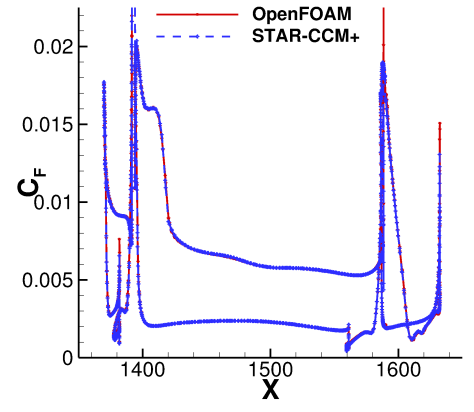

(c)

Fig. 17 Skin-friction coefficient at (a) eta=0.151, (b) 0.329 and (c) 0.552 at $\alpha=8^{0}$ for OpenFOAM and STAR-CCM+. 


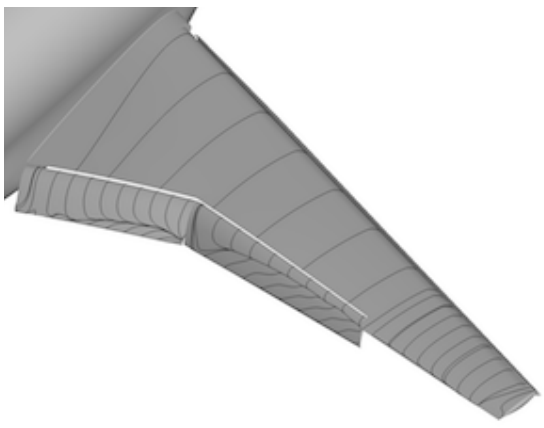

(a)
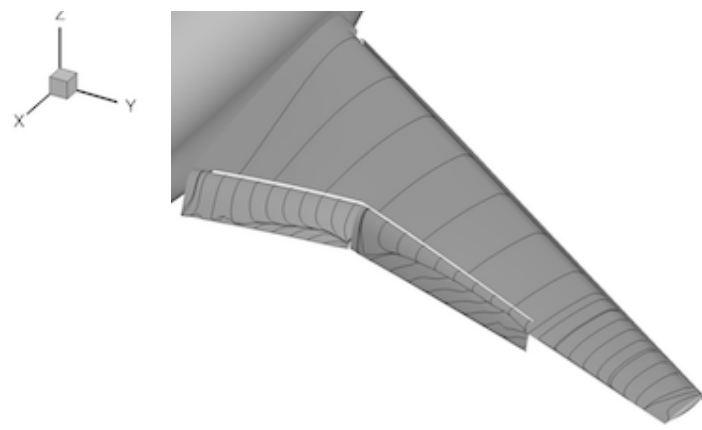

(b)

Fig. 18 Surface streamlines at $\alpha=8^{0}$ for the SA using (a) OpenFOAM and (b) STAR-CCM+.

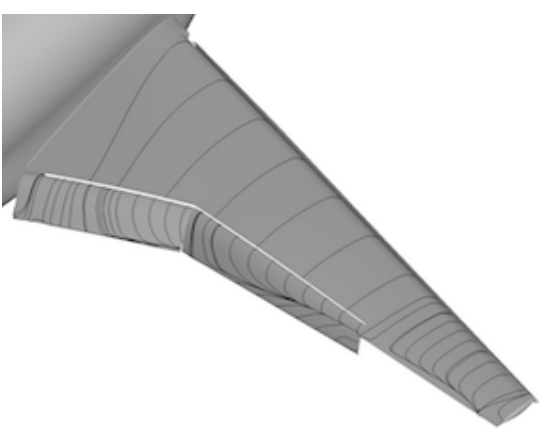

(a)
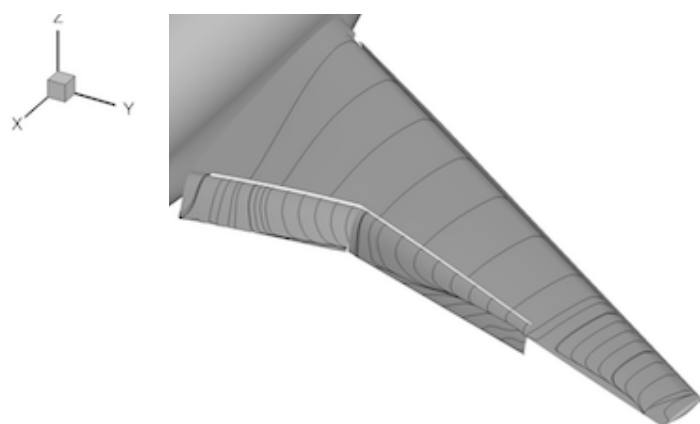

(b)

Fig. 19 Surface streamlines at $\alpha=16^{0}$ for the SA using (a) OpenFOAM and (b) STAR-CCM+.

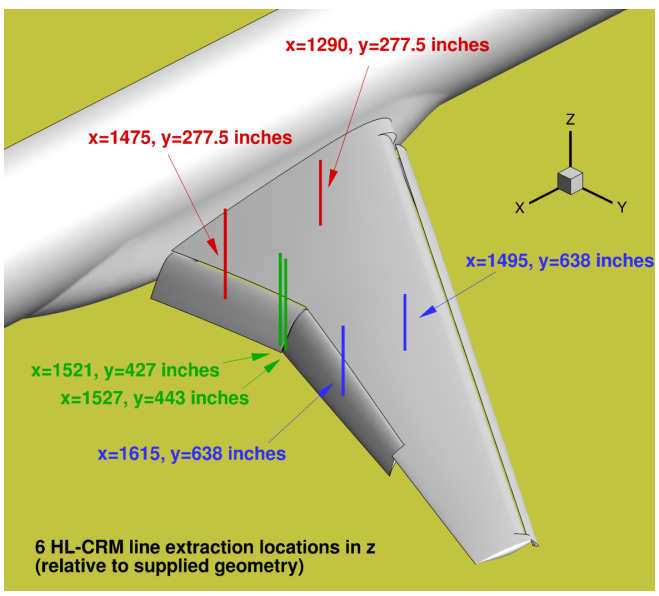

(a)

Fig. 20 Location of velocity profiles for the HL-CRM. 


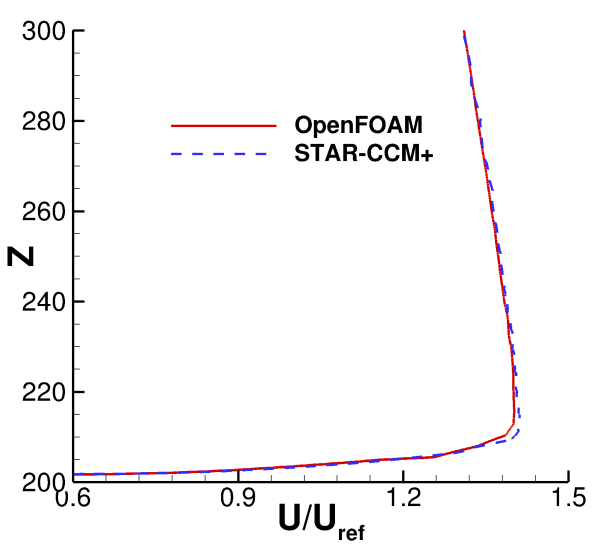

(a)

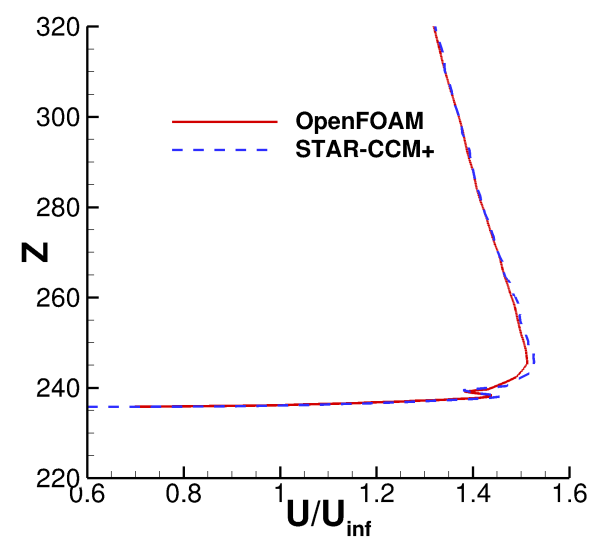

(c)

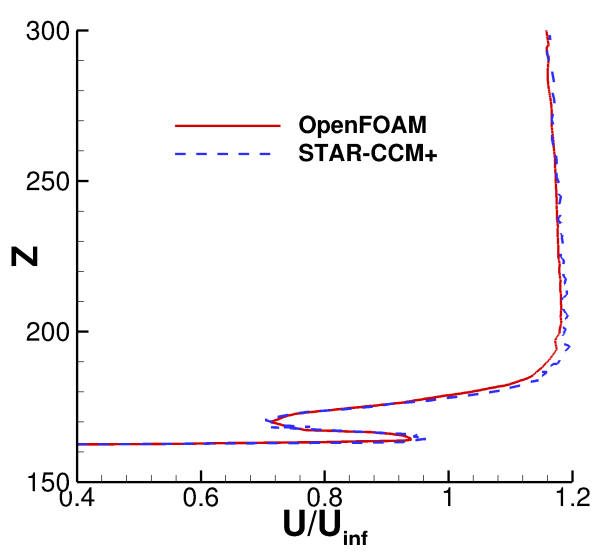

(b)

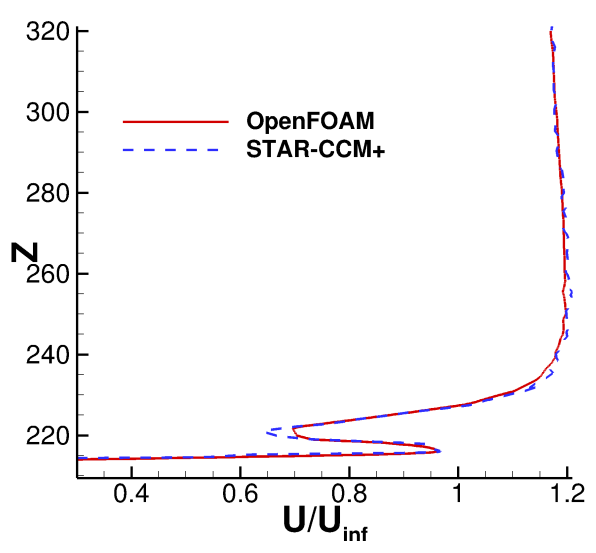

(d)

Fig. 21 Streamwise velocity profiles as shown in Fig. 20 at $\alpha=16^{0}$ for the SA model using OpenFOAM and STAR-CCM+. (a) $\mathrm{x}=1290$ inches, $\mathrm{y}=277.5$ inches, (b) $\mathrm{x}=1475$ inches, $\mathrm{y}=277.5$ inches, (c) $\mathrm{x}=1521$ inches, $\mathrm{y}=427$ inches and (d) $x=1527$ inches, $y=443$ inches

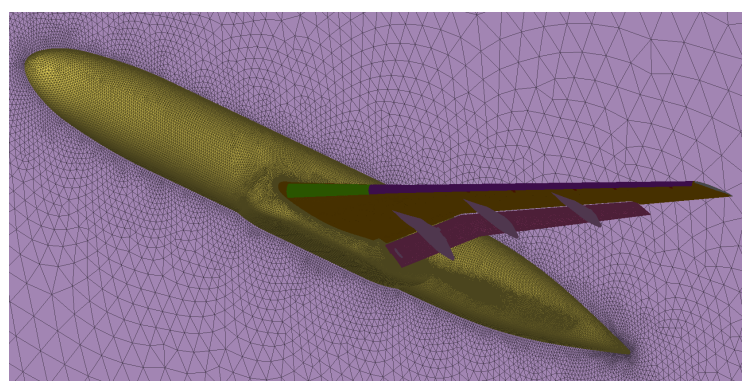

Fig. 22 JSM medium surface mesh.

The differing number of layers around the wing and fuselage was followed in order to ensure a smooth volume ratio transition between the last extruded layer and the pyramid/tetra mesh. Again orthogonality was ensured in the layers 
Table 5 JSM Mesh Properties.

\begin{tabular}{cc}
\hline & Medium w/o nacelle \\
\hline Surface Mesh Face Count & $1.7 \mathrm{M}$ \\
Volume Mesh Cell Count & $109 \mathrm{M}$ \\
Trailing edge rows of elements & 8 \\
Number of layers around Wing/Nacelle/Pylon & $49(57$ fuselage $)$ \\
Layers Growth & 1.16 \\
Layers First Height $(\mathrm{mm})$ & 0.0015 \\
\hline
\end{tabular}

region as shown in Fig. 23 .

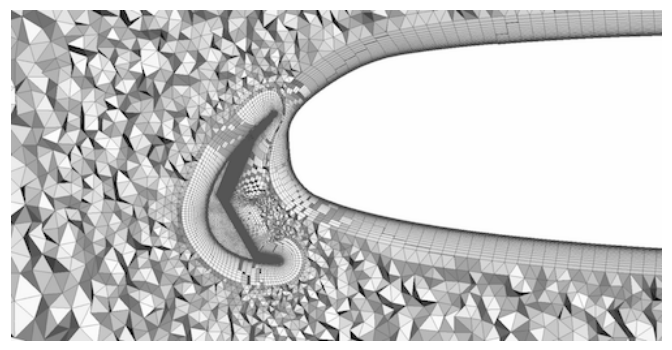

(a)

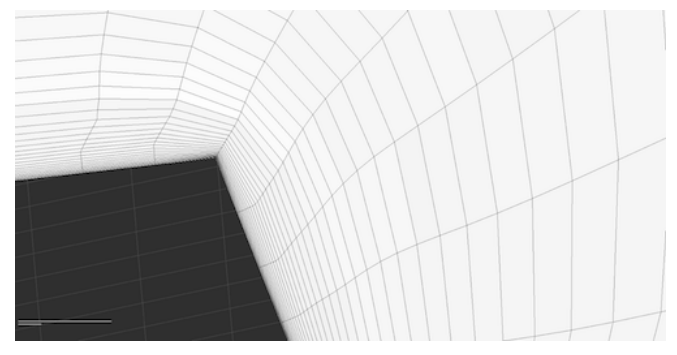

(b)

Fig. 23 (a) Prism layer coverage around the JSM and (b) details of the imposed orthogonality near the wall for better orthogonality and skewness.

\section{Results}

Figure 24 shows the lift and drag curves for OpenFOAM and STAR-CCM+ using the SA model. It can be seen that firstly the agreement between OpenFOAM and STAR-CCM+ is within 3\%, which is in line with the results from the HL-CRM geometry. The lift coefficient is in good agreement with the experimental data during the linear range but over-predicts the lift towards the stall region. The drag coefficient is consistently over-predicted, which was found by all other HLPW participants [26] and may be due to transition or differences in the CFD and Wind-Tunnel models. As with the HL-CRM, the global force coefficients may hide local effects which can cause error cancellation. To investigate this further, a selection of planes across the span of the wing are analysed - as shown in Fig. 25. Figure 26] show the surface streamlines at $\alpha=4.36^{0}$ for the experimental flow visualization paint and the results from OpenFOAM and STAR-CCM+. At this lower angle of attack, the agreement is good between both CFD codes and the experimental data. This is also reflected in Figs. 27 and 28 for the pressure coefficient at planes A-A and H-H, where the CFD closely matches the experimental data. At this low angle of attack, the flow is attached throughout the wing, providing less of a challenge to the SA turbulence model.

Moving to $\alpha=18.59^{\circ}$, the agreement between CFD and experimental data worsens, as shown in Figs. 29, 30 and 
31. The agreement between STAR-CCM+ and OpenFOAM is however close, with little observable differences - which is reflected in the lift and drag coefficients. Using the SA model, both codes over-predict the outboard flow separation and underestimates the beginning of the root stall. This is reflected in the inboard plane A-A in Fig. 30, where the pressure coefficient is over-predicted. At the outboard plane H-H (Fig. 31), an opposite trend is observed where the pressure coefficient is under predicted because of the flow separation. The under and over prediction of flow separation at the inboard and outboard sections cancel out to provide a good prediction of the global lift - a clear example of error cancellation. This was seen by nearly all participants of the 3rd HLPW - even those using more advanced RANS models such as the SA QCR model [29] or full Reynolds stress models [26].

At the stall angle of $\alpha=21.57^{\circ}$, the trends observed at $\alpha=18.59^{\circ}$ continue, as shown in Figs. 32,33 and 34 The discrepancy between the experimental data and CFD is broader at this angle, with the CFD predicting much larger outboard separation whilst also showing little inboard stall. This is clearer in the pressure coefficient plots, where the outboard under-prediction of pressure coefficient due to greater stall is present for both CFD codes. At this angle, the disagreement between between STAR-CCM+ and OpenFOAM grows - although the large scale unsteadiness at this angle likely makes the differences between the numerical schemes greater. The previous HL-CRM case also showed that the differences between both CFD codes reduce as the grid is refined. The JAXA JSM grid was only 100M compared to 236M fine HL-CRM grid and thus it is predicted that a finer grid for this JSM geometry would bring the results for both CFD codes even closer.

Previous work has shown that the standard SA model struggles to capture the anisotropic nature of the turbulence in the wing-root junction [5, 30] - thus it is not surprising that the SA fails to capture this region and has been discussed widely for flows subject to corner separation [31]. The use of a Reynolds stress model [17] or the SA-QCR [29] model was predicted to help this, however none of the 3rd HLPW participants using the SA-QCR or Reynolds stress models captured the inboard separation [26]. In addition participants who used some form of a transition model also did not capture the inboard separation. Thus further research and investigation is needed to understand why CFD is not able to capture the correct stall mechanisms. It may be related to subtle differences in the CFD geometry model and the model used in the experiment, or some transient effect that needs globally time accurate transient methods to capture.

\section{Computational expense}

The previous results have shown that OpenFOAM does largely compare well with the accuracy of a major commercial code when using the same mesh, turbulence model and numerical scheme order. This agrees with the recent work of the first author for a helicopter fuselage [32]. This is encouraging for users of OpenFOAM but this is typically only one aspect of engineering CFD analysis. A major consideration is the robustness and computational cost. 


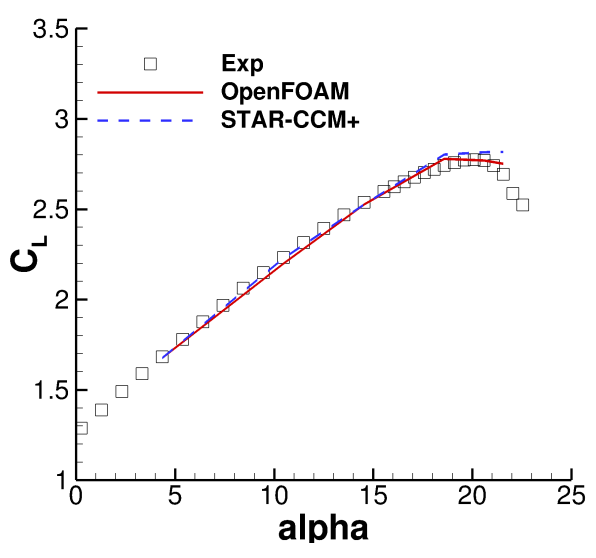

(a)

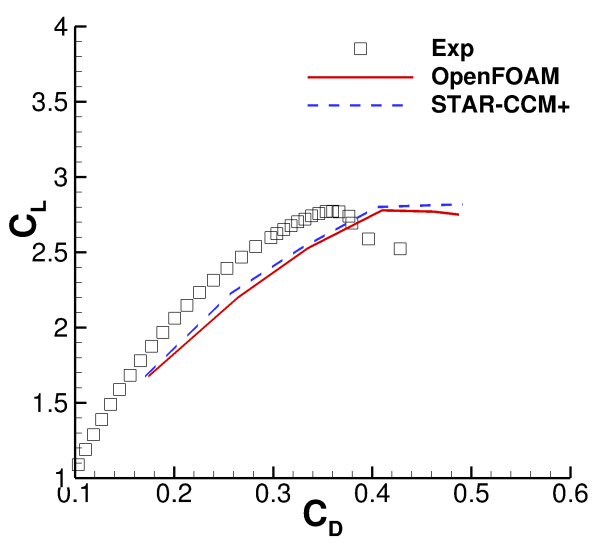

(c)

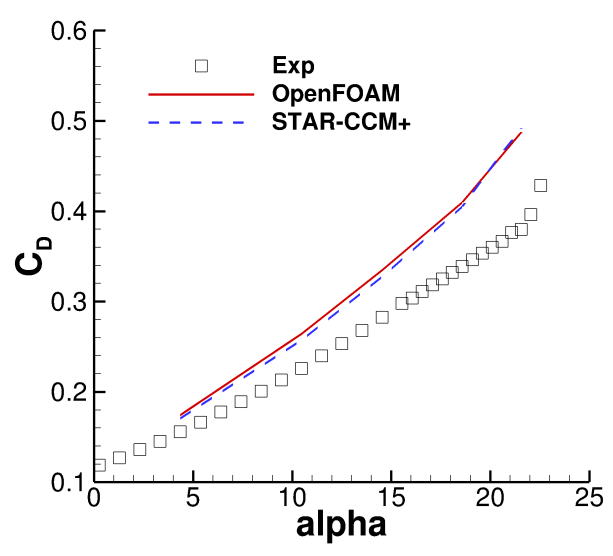

(b)

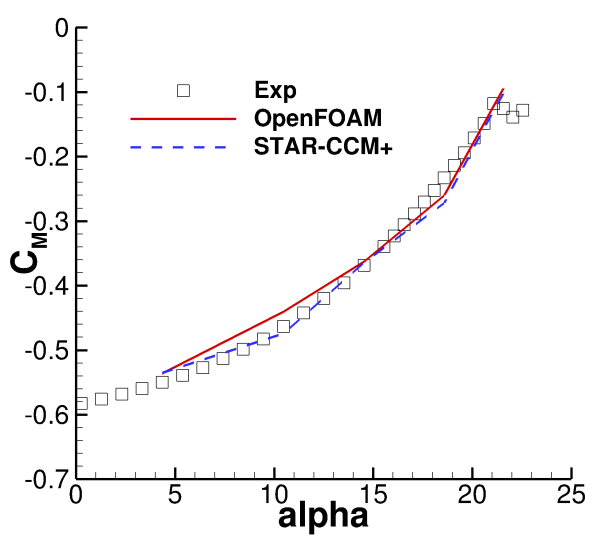

(d)

Fig. 24 (a) Lift Coefficient, (b) Drag Coefficient (c) Lift vs Drag Coefficient and (d) Moment coefficient using the SA model using OpenFOAM and STAR-CCM+.

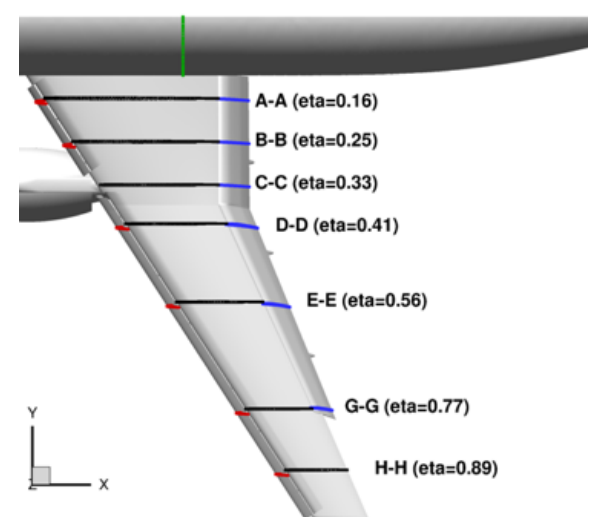

Fig. 25 Schematic of the planes analysed in this section. 


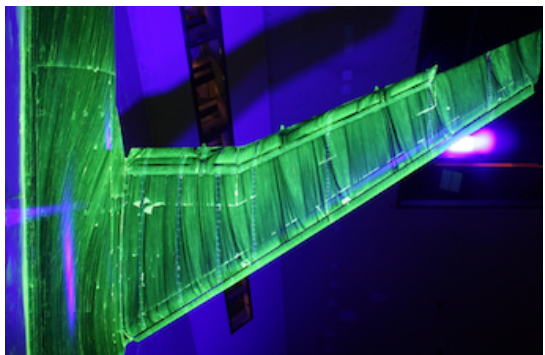

(a)

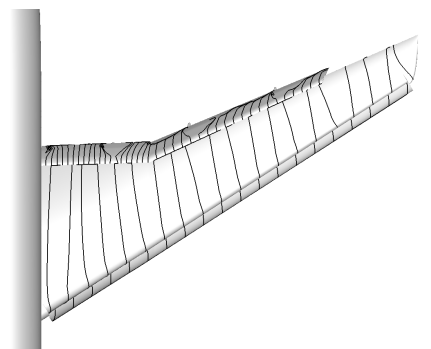

(b)

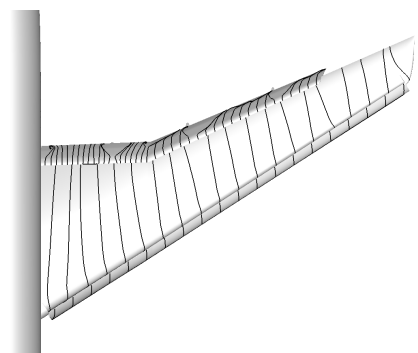

(c)

Fig. 26 Surface streamlines at $\alpha=4.36^{\circ}$ from (a) Experiment and for the SA using (b) OpenFOAM and (c) STAR-CCM+.

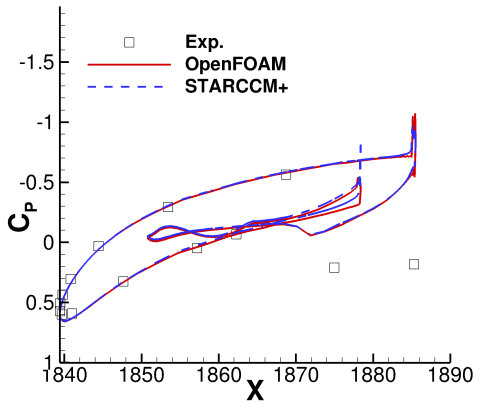

(a)

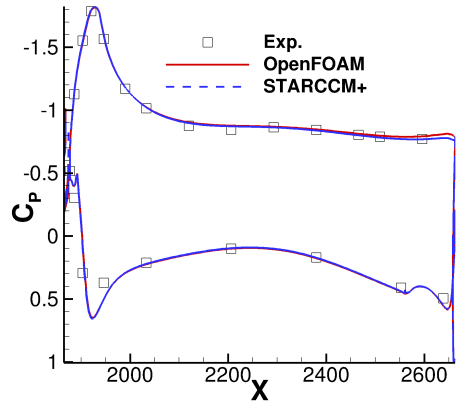

(b)

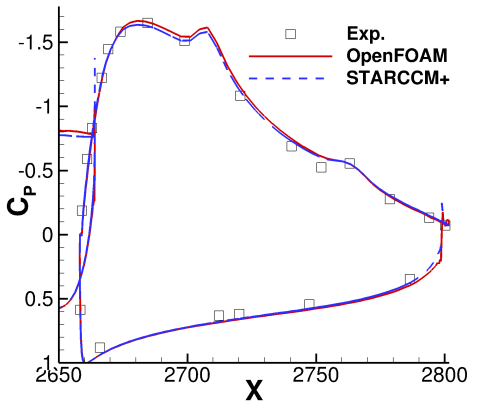

(c)

Fig. 27 Pressure coefficient at Cut A-A at $\alpha=4.36^{\circ}$ on (a) Slat (b) Wing and (c) Flap.

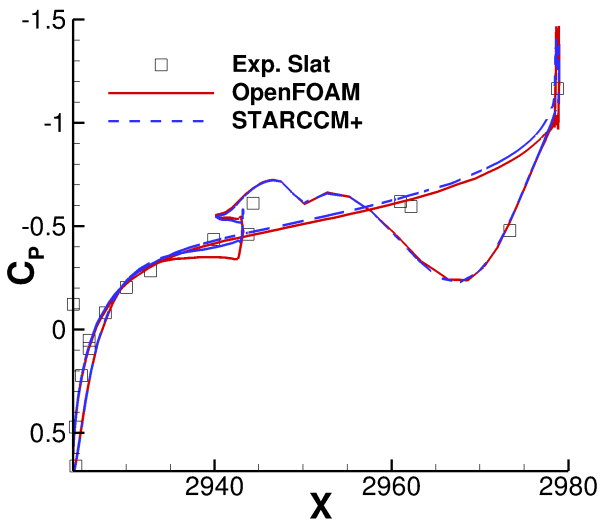

(a)

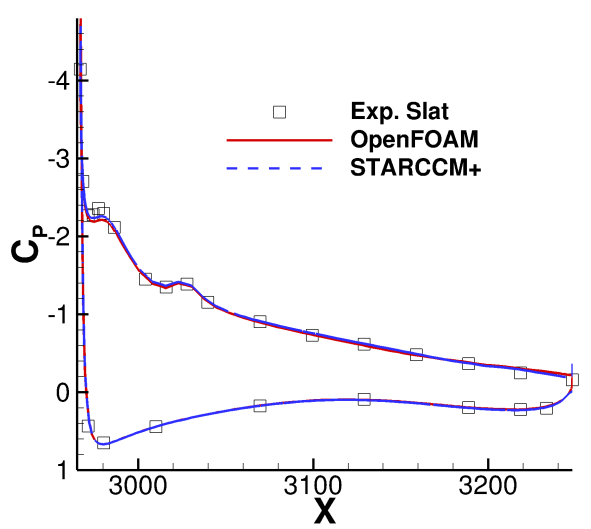

(b)

Fig. 28 Pressure coefficient at Cut H-H at $\alpha=4.36^{\circ}$ on (a) Slat and (b) Wing. 


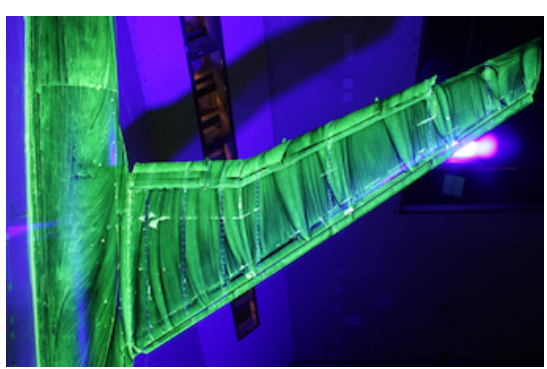

(a)

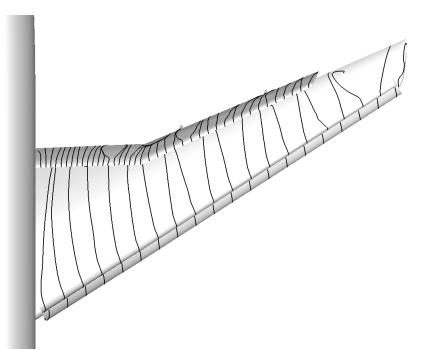

(b)

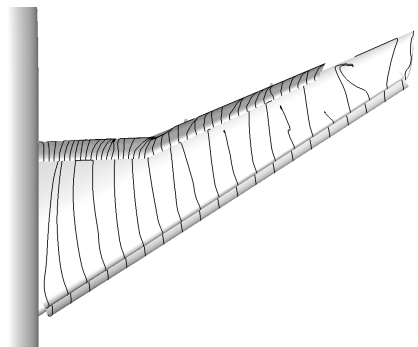

(c)

Fig. 29 Surface streamlines at $\alpha=18.59^{\circ}$ from (a) Experiment and for the SA using (b) OpenFOAM and (c) STAR-CCM+.

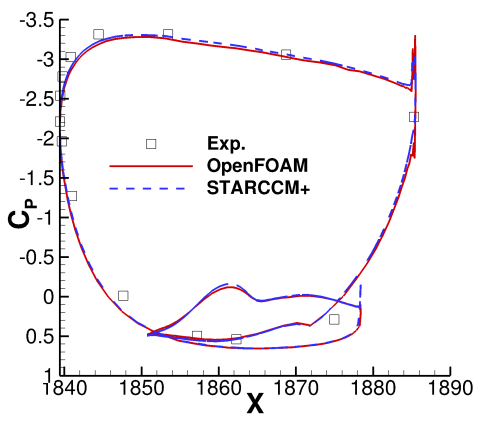

(a)

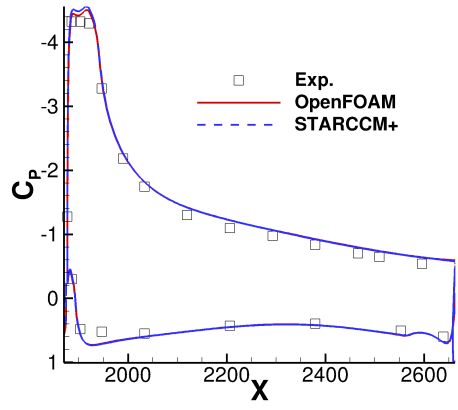

(b)

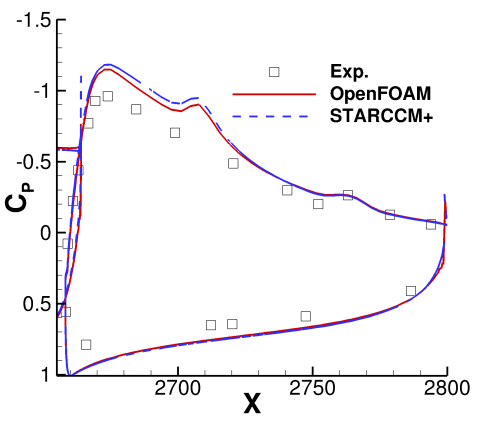

(c)

Fig. 30 Pressure coefficient at Cut A-A at $\alpha=18.58^{\circ}$ on (a) Slat (b) Wing and (c) Flap.

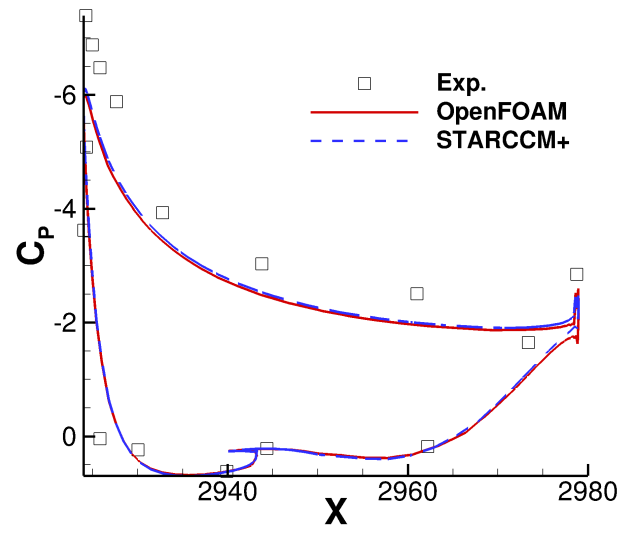

(a)

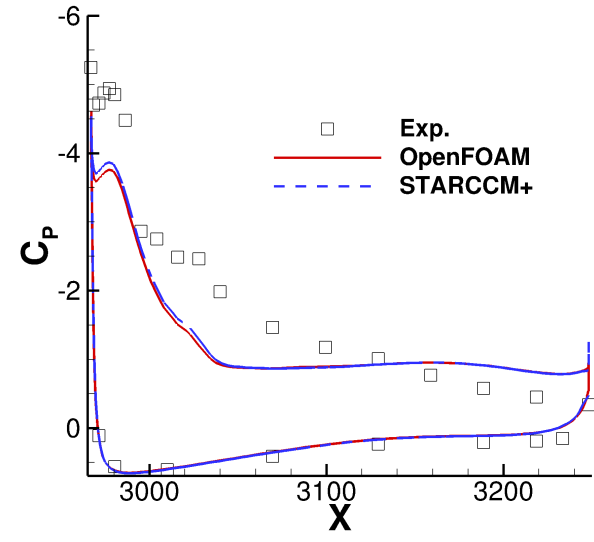

(b)

Fig. 31 Pressure coefficient at Cut H-H at $\alpha=18.58^{\circ}$ on (a) Slat and (b) Wing. 


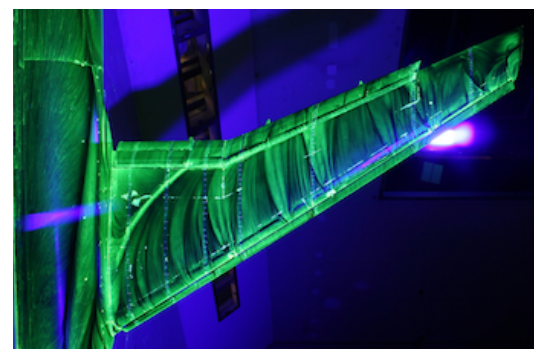

(a)

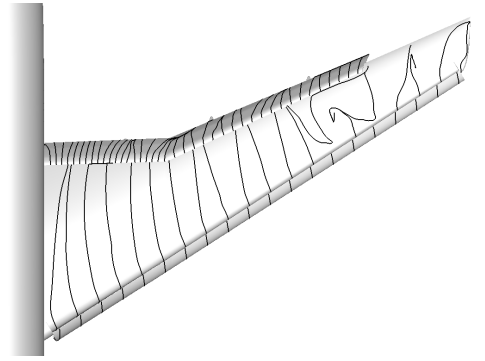

(b)

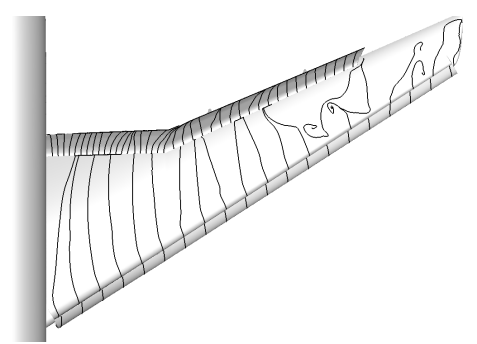

(c)

Fig. 32 Surface streamlines at $\alpha=21.57^{\circ}$ from (a) Experiment and for the SA using (b) OpenFOAM and (c) STAR-CCM+.

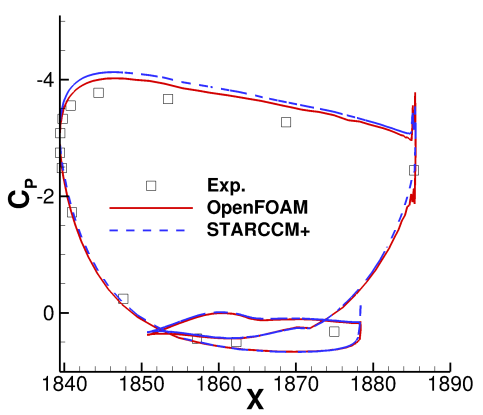

(a)

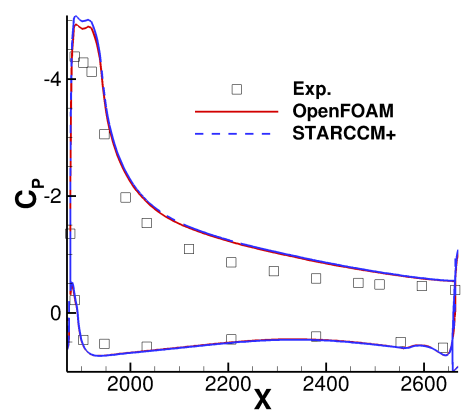

(b)

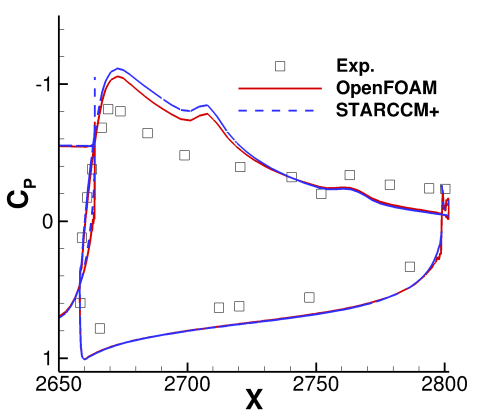

(c)

Fig. 33 Pressure coefficient at Cut A-A at $\alpha=21.57^{\circ}$ on (a) Slat (b) Wing and (c) Flap.

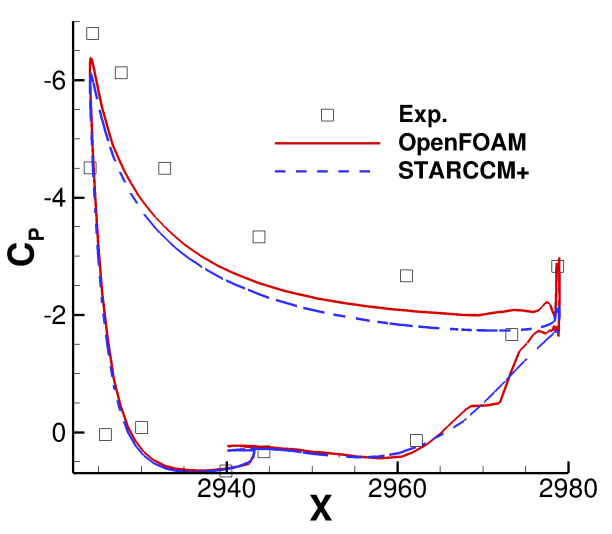

(a)

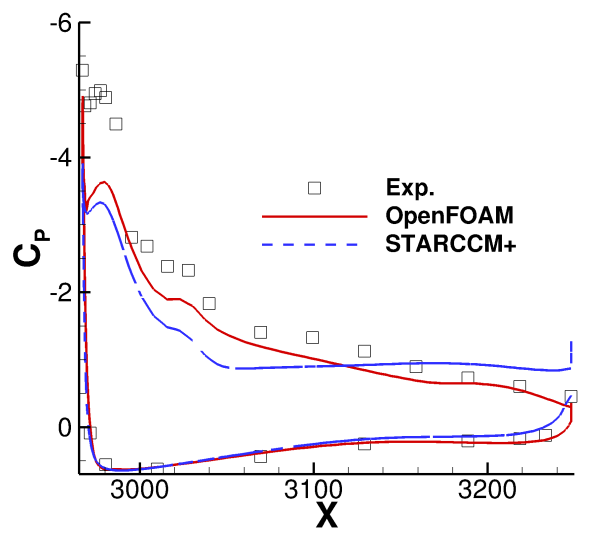

(b)

Fig. 34 Pressure coefficient at Cut H-H at $\alpha=21.57^{\circ}$ on (a) Slat and (b) Wing. 


\section{Robustness and Convergence}

In terms of robustness, STAR-CCM+ was found to need less user input to ensure a stable, converged simulation. Given the correct initial ramping of the CFL number (typically CFL of 10-30 ramped from 0.1 over 500 iterations), the simulation needed no further user input and typically reached convergence of the residuals and forces by 15,000-40,000 iterations (dependent on angle of attack and grid refinement). An example of the force and residual convergence is shown in Figs. 35 and 36(a) for the coarse HL-CRM mesh at 16 degrees AoA. The convergence of the residuals result in at least 4-5 order drop in their magnitude, which is typical for complex geometries and meshes. For the forces it means the lift, drag and moment coefficients are reaching a point where the standard deviation over a given number of iterations was less than $5 e^{-4}$. For the higher angles of attack for the JSM geometry this was not always possible due to the large scale unsteadiness.

For OpenFOAM, trial and error was needed to initially find the most stable setup - this resulted in a limit in the largest local CFL, typically $2-5$. This typically results in convergence from 50,000 iterations to sometimes more than 100,000 for the stalled JSM cases. An example of the force and residual convergence is shown in Figs. 35] and 36(b) for the coarse HL-CRM mesh at 16 AoA. Second order upwind schemes were used for both the momentum and turbulent qualities thus accuracy was prioritised over stability, although typically a solution was required to run initially with 1st order and later switched to 2 nd order which also increased the total number of iterations to achieve convergence. It is not within the scope of this paper to provide an exhaustive analysis of the stability/robustness of OpenFOAM however considerable time was spent to find the optimum configuration.

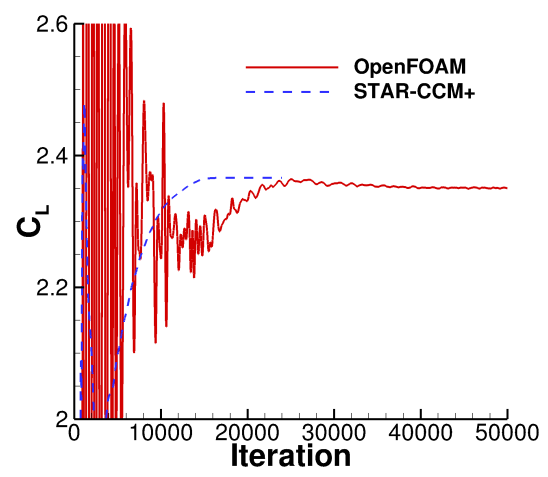

(a)

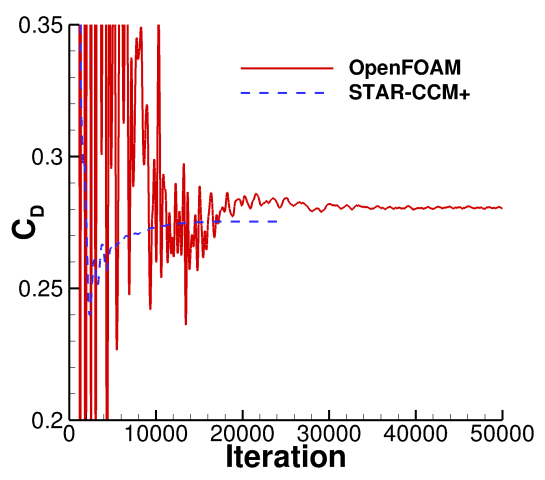

(b)

Fig. 35 Iterative (a) lift coefficient and (b) drag coefficient for HL-CRM coarse mesh at 16 degrees AoA (restarted from 8 degrees AoA solution). 


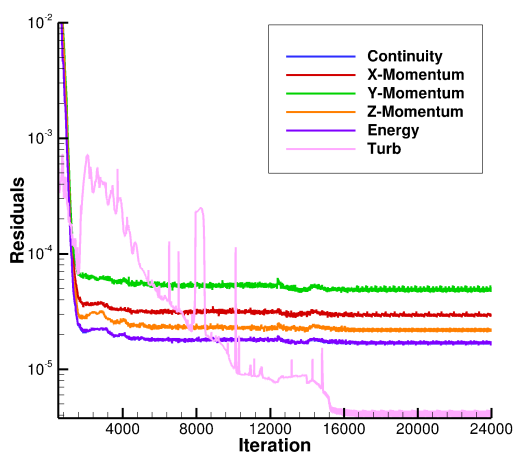

(a)

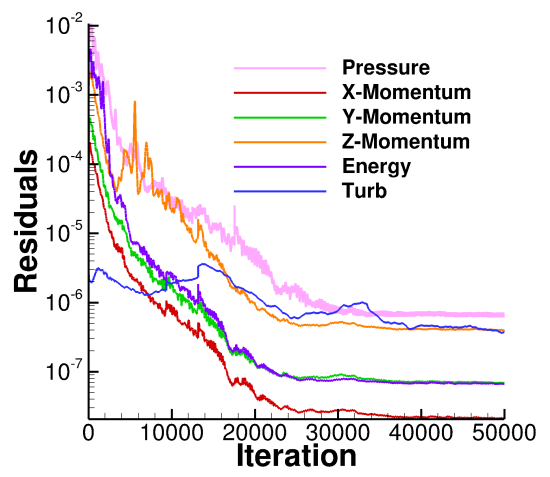

(b)

Fig. 36 Residuals for HL-CRM coarse mesh at 16 AoA (restarted from 8 AoA solution) for (a) STAR-CCM+ and (b) OpenFOAM.

\section{Computational expense}

Simulations were conducted on the University of Oxford Advanced Research Computing service (ARC) as well as the UK National Supercomputer ARCHER. Figure 37 indicates the strong scaling of OpenFOAM for production runs on the ARCHER system. It can be seen that the close to linear scaling is achieved down to 50,000 cells per core ( 5000 cores for fine HL-CRM mesh). Unfortunately at the time of access to ARCHER, STAR-CCM+ was not available on the system and thus scaling tests were not possible.

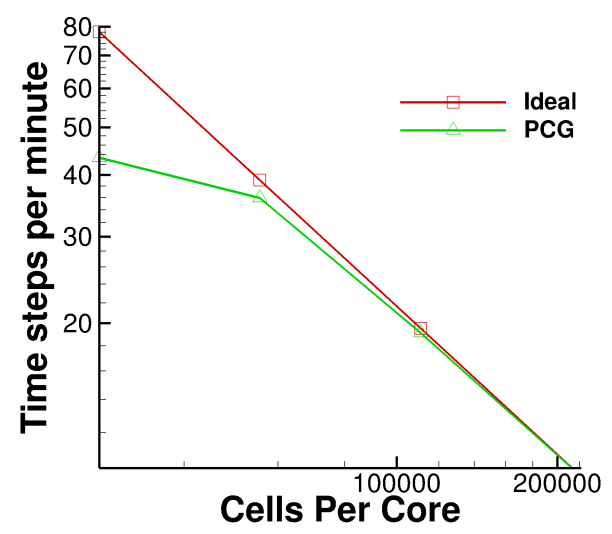

Fig. 37 Scaling of OpenFOAM on the UK national supercomputer - ARCHER.

To compare the computational efficiency of STAR-CCM+ and OpenFOAM, both codes were compiled in double precision on the Oxford ARC system which contains dual Intel E5-2640v3 Haswell CPU nodes which have 16 cores per node and $64 \mathrm{~Gb}$ of memory. The coarse HL-CRM mesh was used for this comparison at 16 degrees AoA and run for 300 iterations three times to obtain an average time per iteration. These numbers do not reflect the optimum speed 
of each code, which would require both specific compiler optimisation work and a detailed assessment of individual solver convergence tolerance settings (which impact number of inner iterations for each solver). These numbers are however added to help to provide an overall view of the differences in accuracy and computational efficiency between OpenFOAM and STAR-CCM+ on a typical HPC system. At higher angles of attack more iterations were typically required with a lower CFL number - conversely for lower angles of attack higher CFL numbers could be used resulting in fewer iterations, which was true of both OpenFOAM and STAR-CCM+.

Table 6 Computational expense study for HL-CRM coarse mesh at 16 AoA.

\begin{tabular}{cccccc}
\hline Code & Cells & Cores & Iteration Time $(\mathrm{s})$ & Iterations & Total time $(\mathrm{hrs})$ \\
\hline OpenFOAM & $89 \times 10^{6}$ & 320 & 6.8 & 55,000 & 103 \\
STAR-CCM+ & $89 \times 10^{6}$ & 320 & 9.5 & 24,000 & 63.3 \\
\hline
\end{tabular}

It is clear from Table 6 that whilst OpenFOAM is approximately $30 \%$ faster per iteration, the biggest difference is the number of iterations required to reach convergence in the forces and residuals. It is no surprise that the coupled implicit density based scheme within STAR-CCM+ is able to achieve convergence in fewer iterations compared to the implicit segregated pressure based approach within OpenFOAM. This provides important evidence needed to encourage the OpenFOAM community to develop a robust coupled implicit density based scheme for the official version of OpenFOAM so that these large-scale complex aerospace applications can be undertaken with greater computational efficiency.

\section{Conclusions}

This paper presents an assessment of a popular open-source code, OpenFOAM for compressible high-lift aircraft flows. A range of cases are investigated including a zero-pressure gradient flat plate, NACA0012 airfoil at varying angles of attack, the DSMA661 airfoil, NASA high-lift common-research model and a JAXA JSM high-lift aircraft model. For all cases the same mesh and turbulence is also simulated in the commercial code STAR-CCM+ to provide a benchmark solution from a widely used CFD code. For the HL-CRM and JAXA JSM models the lift and drag coefficients from OpenFOAM are within 3\% of the STAR-CCM+ solution. For the JAXA JSM case with experimental data the CFD simulations agree well with the experimental at pre-stall AoA but for post-stall whilst the global loads are well predicted, the local separation is not well predicted and the global force agreement is due to error cancellation. This result is however in line with expectations for the SA model with no corrections for curvature or corner flows. It is noted that even with great care to generate high quality unstructured hybrid prismatic-tetrahedral meshes using the commercial tool ANSA, the robustness of the standard version of OpenFOAM 4.1 was inferior to STAR-CCM+ and typically required twice as long to reach a satisfactory force convergence largely due to the inability to run as higher Courant numbers as STAR-CCM+. It is hoped that this work can be inspire further development of compressible 
methods within OpenFOAM to improve the computational efficiency and robustness.

\section{Acknowledgements}

The authors gratefully acknowledge computational support from EPSRC for UK's National HPC Facility, ARCHER. The authors would also like to acknowledge the use of the University of Oxford Advanced Research Computing (ARC) facility in carrying out this work. http://dx.doi.org/10.5281/zenodo.22558 .

\section{References}

[1] Spalart, P. R., and Venkatakrishnan, V., "On the role and challenges of CFD in the aerospace industry," The Aeronautical Journal, Vol. 120, No. 1223, 2016, pp. 209-232. doi:10.1017/aer.2015.10, URL/http://www.journals.cambridge.org/ abstract_S000192401500010X.

[2] Slotnick, J., Khodadoust, A., Alonso, J., Darmofal, D., Gropp, W., Lurie, E., and Mavriplis, D., “CFD Vision 2030 Study: A Path to Revolutionary Computational Aerosciences," Nasa Cr-2014-21878, , No. March, 2014. URL http: //ntrs.nasa.gov/search.jsp?R=20140003093

[3] Menzies, K., "Delivering better power: the role of simulation in reducing the environmental impact of aircraft engines." Philosophical transactions. Series A, Mathematical, physical, and engineering sciences, Vol. 372, No. 2022, 2014. doi:10. 1098/rsta.2013.0316, URL http://www. scopus. com/inward/record.url?eid=2-s2.0-84904302078\&partnerID= tZ0tx3y1

[4] Abbas-Bayoumi, A., and Becker, K., “An industrial view on numerical simulation for aircraft aerodynamic design,” Journal of Mathematics in Industry, Vol. 1, No. 1, 2011, p. 10. doi:10.1186/2190-5983-1-10.

[5] Rumsey, C. L., and Slotnick, J. P., “Overview and Summary of the Second AIAA High Lift Prediction Workshop,” 52nd Aerospace Sciences Meeting, American Institute of Aeronautics and Astronautics, Reston, Virginia, 2014. doi:10.2514/6.2014-0747, URL http://dx.doi.org/10.2514/6.2014-0747http://arc.aiaa.org/doi/abs/10.2514/6.2014-0747

[6] Spalart, P. R., Jou, W. H., Strelets, M., and Allmaras, S. R., "Comments on the feasibility of LES for wings and on a hybrid, RANS/L ES approach,” Advances in DNS/LES, Proceedings of 1st AFOSR International Conference on DNS/LES, Vol. 1, 1997, pp. $137-147$.

[7] Ashton, N., West, A., and Mendonça, F., "Flow Dynamics Past a 30P30N Three-Element Airfoil Using Improved Delayed Detached-Eddy Simulation," AIAA Journal, 2016, pp. 1-10. doi:10.2514/1.J054521, URL http://arc . aiaa.org/doi/10. $2514 / 1 . J 054521$

[8] Economon, T. D., Palacios, F., Copeland, S. R., Lukaczyk, T. W., and Alonso, J. J., "SU2: An Open-Source Suite for Multiphysics Simulation and Design,” AIAA Journal, Vol. 54, No. 3, 2016, pp. 828-846. doi:10.2514/1.J053813, URL http://arc.aiaa.org/doi/10.2514/1.J053813 
[9] Archambeau, F., Mechitoua, N., and Sakiz, M., "A finite volume method for the computation of turbulent incompressible flows industrial applications.” Int. J. Finite Volumes, Vol. 1, 2004, pp. 1-62.

[10] Jasak, H., "Error analysis and estimation for the finite volume method with applications to fluid flows." Ph.D. thesis, 1996. URL http://ethos.bl.uk/OrderDetails.do?uin=uk.bl.ethos. 311802

[11] Spalart, P. R., and Allmaras, S. R., “A one-equation turbulence model for aerodynamic flows," La Recherche Aerospatiale, Vol. 1, 1994, pp. 5-21.

[12] Weller, H. G., and Tabor, G., "A tensorial approach to computational continuum mechanics using object-oriented techniques," Computers in Physics, Vol. 12, No. 6, 1998, pp. 620-631. doi:10.1063/1.168744.

[13] Issa, R. I., "Solution of the implicitly discretised fluid flow equations by operator-splitting," Journal of Computational Physics, Vol. 62, No. 1, 1986, pp. 40-65. doi:10.1016/0021-9991(86)90099-9.

[14] Nikaido, B. E., Murman, S. M., and Garcia, J., "OpenFOAM Simulations of Atmospheric-Entry Capsules in the Subsonic Regime," 53rd AIAA Aerospace Sciences Meeting, , No. January, 2015, pp. 1-14. doi:10.2514/6.2015-0313, URL http: //arc.aiaa.org/doi/abs/10.2514/6.2015-0313

[15] Robertson, E., Choudhury, V., Bhushan, S., and Walters, D. K., "Validation of OpenFOAM numerical methods and turbulence models for incompressible bluff body flows," Computers and Fluids, Vol. 123, 2015, pp. 122-145. doi:10.1016/j.compfluid. 2015.09.010, URL http://dx.doi.org/10.1016/j.compfluid.2015.09.010

[16] Gomez, S., Graves, B. J., and Poroseva, S. V., "On the Accuracy of RANS Simulations of 2D Boundary Layers with OpenFOAM," AIAA Journal, , No. June, 2014, pp. 1-9. doi:doi:10.2514/6.2014-2087.

[17] Stoellinger, M. K., Roy, R., and Ashton, N., “Application of an Elliptic Blending Reynolds Stress Model in Attached and Separated flows (Invited)," 22nd AIAA Computational Fluid Dynamics Conference, American Institute of Aeronautics and Astronautics, Reston, Virginia, 2015, pp. 1-12. doi:10.2514/6.2015-2926, URL http://arc.aiaa.org/doi/10.2514/6.2015-2926

[18] Tucker, P., Unsteady Computational Fluid Dynamics in Aeronautics, Fluid Mechanics and Its Applications, Vol. 104, Springer Netherlands, Dordrecht, 2014. doi:10.1007/978-94-007-7049-2, URL http://link.springer .com/10.1007/978-94007-7049-2.

[19] Tucker, P. G., Rumsey, C. L., Spalart, P. R., Bartels, R. E., and Biedron, R. T., "Computations of wall distances based on differential equations," AIAA Journal, Vol. 43, No. 3, 2005, pp. 539-549. doi:10.2514/1.8626, URL http://www . scopus. com/inward/record. url?eid=2-s2.0-15944377925\&partnerID=40\&md5=a40f830889eb58b4d527d90f1737dcf9

[20] Kareem, A., Spence, S. M., and Wei, D., "Turbulence Model Verification and Validation in an Open Source Environment," Progress in Computational Fluid Dynamics, An International Journal, Vol. 1, No. 1, 2016, p. 1. doi:10.1504/PCFD.2016. 10001448, URL http://wWw.inderscience.com/link.php?id=10001448

[21] Menter, F. R., “Improved two-equation k-\$lomega\$ Turbulence models for aerodynamic flows,” Tech. rep., NASA, 1992. 
[22] “3rd AIAA CFD High Lift Prediction Workshop,” http://hiliftpw.larc.nasa.gov, 2017. URL https://hiliftpw.larc.nasa. gov.

[23] Vassberg, J., Dehaan, M., Rivers, M., and Wahls, R., "Development of a Common Research Model for Applied CFD Validation Studies," 26th AIAA Applied Aerodynamics Conference, American Institute of Aeronautics and Astronautics, Reston, Virigina, 2008, pp. 1-22. doi:10.2514/6.2008-6919, URL http://arc.aiaa.org/doi/10.2514/6.2008-6919

[24] Ashton, N., Unterlechner, P., and Blacha, T., "Assessing the Sensitivity of Hybrid RANS-LES Simulations to Mesh Resolution, Numerical Schemes and Turbulence Modelling within an Industrial CFD Process," SAE Technical Papers, Vol. 2018-April, 2018. doi:10.4271/2018-01-0709.

[25] Skaperdas, V., and Ashton, N., "Development of high-quality hybrid unstructured meshes for the GMGW-1 workshop using ANSA,” 2018 AIAA Aerospace Sciences Meeting, American Institute of Aeronautics and Astronautics, Reston, Virginia, 2018. doi:10.2514/6.2018-0660, URL https://arc.aiaa.org/doi/10.2514/6.2018-0660

[26] Rumsey, C. L., Slotnick, J. P., and Sclafani, A. J., "Overview and Summary of the Third AIAA High Lift Prediction Workshop," 2018 AIAA Aerospace Sciences Meeting, American Institute of Aeronautics and Astronautics, Reston, Virginia, 2018, pp. 1-33. doi:10.2514/6.2018-1258, URL/https://ntrs.nasa.gov/archive/nasa/casi.ntrs.nasa.gov/20140011924 pdfhttps://arc.aiaa.org/doi/10.2514/6.2018-1258.

[27] Ashton, N., Denison, M., and Zastawny, M., "3rd High-Lift Workshop Summary Paper - OpenFOAM, STAR-CCM+ \&amp; LAVA simulations on Unstructured Grids,” 2018 AIAA Aerospace Sciences Meeting, American Institute of Aeronautics and Astronautics, Reston, Virginia, 2018, pp. 1-25. doi:10.2514/6.2018-1253, URL https://arc.aiaa.org/doi/10.2514/6. $2018-1253$

[28] Yokokawa, Y., Murayama, M., Uchida, H., Tanaka, K., Ito, T., and Yamamoto, K., “Aerodynamic influence of a half-span model installation for high-lift configuration experiment," 48th AIAA Aerospace Sciences Meeting Including the New Horizons Forum and Aerospace Exposition, , No. January, 2010, pp. 1-17. URL http://www. scopus . com/inward/record.url?eid=2s2.0-78649869623\&partnerID=40\&md5=d18e329e728b362ee827a9e6f0532224

[29] Spalart, P., "Strategies for turbulence modelling and simulations," International Journal of Heat and Fluid Flow, Vol. 21, No. 3, 2000, pp. 252-263. doi:10.1016/S0142-727X(00)00007-2, URL http://linkinghub.elsevier.com/retrieve/pii/ S0142727X00000072

[30] Spalart, P. R., "Progress in Aerospace Sciences Philosophies and fallacies in turbulence modeling," Progress in Aerospace Sciences, 2015, pp. 1-15. doi:10.1016/j.paerosci.2014.12.004, URL http://dx.doi.org/10.1016/j.paerosci.2014. 12.004

[31] Apsley, D. D., and Leschziner, M. A., “Advanced Turbulence Modelling of Separated Flow in a Diffuser,” Flow, Turbulence and Combustion, Vol. 63, 2000, pp. 81-112. 
[32] Ashton, N., Fuchs, M., Mockett, C., and Buda, B., "EC135 Helicopter Fuselage," Go4Hybrid: Grey Area Mitigation for Hybrid RANS-LES Methods, Notes on Numerical Fluid Mechanics and Multidisciplinary Design, Vol. 134, edited by C. Mockett, W. Haase, and D. Schwamborn, Springer International Publishing, Cham, 2018, pp. 2013-2015. doi: 10.1007/978-3-319-52995-0. 